Motion tracking-enhanced MART for tomographic PIV

This article has been downloaded from IOPscience. Please scroll down to see the full text article.

2010 Meas. Sci. Technol. 21035401

(http://iopscience.iop.org/0957-0233/21/3/035401)

View the table of contents for this issue, or go to the journal homepage for more

Download details:

IP Address: 131.180.130.109

The article was downloaded on 07/11/2011 at 09:40

Please note that terms and conditions apply. 


\title{
Motion tracking-enhanced MART for tomographic PIV
}

\author{
Matteo Novara $^{1}$, Kees Joost Batenburg ${ }^{2}$ and Fulvio Scarano ${ }^{1}$ \\ ${ }^{1}$ Aerospace Engineering Department, Delft University of Technology, 2629 HS, The Netherlands \\ ${ }^{2}$ Vision Lab, University of Antwerp (CDE), Universiteitsplein 1, B-2610 Wilrijk, Belgium \\ E-mail: M.Novara@tudelft.nl
}

Received 23 October 2009, in final form 1 December 2009

Published 25 January 2010

Online at stacks.iop.org/MST/21/035401

\begin{abstract}
A novel technique to increase the accuracy of multiplicative algebraic reconstruction technique (MART) reconstruction from tomographic particle image velocimetry (PIV) recordings at higher seeding density than currently possible is presented. The motion tracking enhancement (MTE) method is based on the combined utilization of images from two or more exposures to enhance the reconstruction of individual intensity fields. The working principle is first introduced qualitatively, and the mathematical background is given that explains how the MART reconstruction can be improved on the basis of an improved first guess object obtained from the combination of non-simultaneous views reduced to the same time instant deforming the 3D objects by an estimate of the particle motion field. The performances of MTE are quantitatively evaluated by numerical simulation of the imaging, reconstruction and image correlation processes. The cases of two or more exposures obtained from time-resolved experiments are considered. The iterative application of MTE appears to significantly improve the reconstruction quality, first by decreasing the intensity of the ghost images and second, by increasing the intensity and the reconstruction precision for the actual particles. Based on computer simulations, the maximum imaged seeding density that can be dealt with is tripled with respect to the MART analysis applied to a single exposure. The analysis also illustrates that the maximum effect of the MTE method is comparable to that of doubling the number of cameras in the tomographic system. Experiments performed on a transitional jet at $R e=$ 5000 apply the MTE method to double-frame recordings. The velocity measurement precision is increased for a system with fewer views (two or three cameras compared with four cameras). The ghost particles' intensity is also visibly reduced although to a lesser extent with respect to the computer simulations. The velocity and vorticity field obtained from a three-camera reconstruction with MTE are equivalent to that from a four-camera analysis. Possible variants of the MTE algorithm are investigated based on a first guess obtained by average or by product of pseudo-simultaneous objects (PSO), which potentially offer a higher convergence rate.
\end{abstract}

Keywords: tomographic PIV, MART, motion tracking enhancement (MTE)

\section{Introduction}

The measurement of unsteady three-dimensional flows and most notably turbulence is highly relevant in many engineering applications. However, the inherent range of coexisting spatial and temporal scales poses a formidable challenge to measurement techniques, which has led to the development of several 3D measurement methods based on particle image velocimetry. Among the most studied are holographic PIV
(HPIV, Hinsch 2002) based on the interference pattern of a reference light beam and scattered light by particles to determine particle locations in depth; digital holographic PIV (Coëtmellec et al 2001) replaces the photographic plate with a CCD sensor; scanning PIV (Brücker 1995) based on the fast scan of a volume along the depth direction with a laser sheet; 3D-particle tracking velocimetry (Maas et al 1993); the DDPIV method based on particle defocusing (Pereira and Gharib 2001); and the recently established 
tomographic PIV (Tomo-PIV, Elsinga et al 2006a) based on the concept of tomographic reconstruction from multiple views of the particle tracers field. Compared to the previously mentioned techniques, Tomo-PIV allows the achievement of instantaneous fully 3D velocity field measurements, with relatively high density of particle images. The most important improvement offered by tomographic PIV (TomoPIV) is in terms of the maximum number of particle tracers that can be dealt with by the $3 \mathrm{D}$ reconstruction algorithm based on the multiplicative algebraic reconstruction technique (MART, Herman and Lent 1976). Experiments performed in the last 2 years showed that a seeding density of 30000 particles/megapixel can be accurately reconstructed and their motion faithfully tracked by spatial cross-correlation algorithms (Scarano et al 2006, Elsinga et al 2008). This is why this technique is a good candidate for the analysis of turbulent flows and has been broadly adopted for turbulent shear layers analysis (Schröder et al 2008, Scarano and Poelma 2009).

Among the drawbacks of this technique are its strong dependence on high precision calibration and the limitations on accuracy caused by optical aberrations. The former issue can be solved by use of the volume self-calibration algorithm (Wieneke 2008), instead the latter aspect has not been addressed yet. Another important aspect is the reconstruction accuracy dependence upon the concentration of particle images collected on the imagers. When the source density exceeds the typical level mentioned above and for a given number of viewing cameras, the reconstructed object accuracy drops below acceptable levels, also affecting the reliability of the cross-correlation analysis. As for any other 3D technique, tomographic PIV lacks spatial resolution when compared to planar PIV due to the limited number of particles in the measurement domain, which motivates sustained efforts to increase the allowed seeding density. Currently, the only solution to the problem is the use of large-format sensors or an increase in the number of simultaneous views. In both cases, the tomographic system increases in cost and complexity.

The high computational cost, whereby a fraction of an hour is needed to analyze one tomographic snapshot returning the instantaneous 3D velocity vector field, justifies the number of studies devoted to increase the computational efficiency of the reconstruction and of the particle motion analysis. Based on the iterative structure of the tomographic reconstruction algorithm, Worth and Nickels (2008) achieved a reduction of the computational efforts using a multiplicative first guess (MFG) instead of the uniform first guess initially proposed by Elsinga et al (2006a). This approach has been brought forward by a sparse object reconstruction and the cross-correlation technique recently proposed by Atkinson and Soria (2009). An acceleration over more than one order of magnitude is obtained introducing a new initial guess estimate based on multiple lineof-sight (MLOS) matched with a simultaneous correction of the solution (simultaneous algebraic reconstruction technique, SART) without compromising the reconstruction accuracy in comparison to MART.

With the exception of the optimization studies of the MART algorithm for Tomo-PIV, only a few works are available to date that aim at further improving the accuracy of tomographic reconstruction of particle images. Petra et al (2009) investigated in their work an algorithm based on sparsity maximization and that strongly outperformed MART according to numerical simulations. Further studies are however needed to verify the applicability to real experimental conditions of optimization methods, where good a priori knowledge of the object to be reconstructed is required.

The present study proposes a novel digital technique to increase the accuracy of MART reconstruction for a given set of measurements and to allow the reliable reconstruction of images with higher seeding density than currently achievable with the MART algorithm alone. The work is therefore focused on the enhancement of the MART reconstruction technique by means of an algorithm that takes into account the non-simultaneous views from at least two exposures. Because the method is based on the utilization of a particle motion field estimate, the only possible approach is by iteration, whereby an estimate of the particle motion is obtained applying the $3 \mathrm{D}$ cross-correlation analysis commonly referred to in the literature.

The paper gives a few introductory concepts of tomographic reconstruction in the next section along with a description of the working principles of the motion tracking enhancement (MTE) technique. The performances of such an approach are compared with MART by means of numerical simulations. An experimental assessment of this technique is performed on laboratory experiments performed on a transitional jet, where the results obtained by MART with and without MTE are compared. The study also compares two possible variants of the MTE-MART based on sum and product of the pseudo-simultaneous objects.

\section{Tomographic reconstruction}

For the purpose of the present study, the problem of tomography is regarded as the reconstruction of a threedimensional object from a set of its two-dimensional projections. In Tomo-PIV, the $2 \mathrm{D}$ projections are the diffraction-limited images of the particle tracers. This implies that the reconstructed three-dimensional object resolution is also limited by diffraction effects. The projections, line integrals of the unknown intensity distribution along the lines of sight (LOS), are the images recorded by the cameras composing the tomographic imaging system. A broad classification of reconstruction methods (Mishra et al 1999) leads to three different groups of tomographic algorithms: the series expansion methods, the optimization methods and the transform methods. Optimization methods require a good knowledge of the object to be reconstructed, while transform methods are suitable in those cases where a large amount of projection data is available (e.g. medical tomography). This is not the case for Tomo-PIV, which is characterized by a small number of projections (typically three to six) within a limited viewing angle. These features classify the reconstruction problem as limited-data tomography. It was shown that the series expansion methods are the most suitable for these applications, and among them, the MART algorithm 
is well suited for sharp objects (Verhoeven 1993) and has been extensively validated for PIV (Elsinga et al 2006b).

\subsection{Introductory concepts}

The MART algorithm (Herman and Lent 1976) iteratively solves a set of linear equations modeling the tomographic system. In Tomo-PIV, the three-dimensional object intensity $E(X, Y, Z)$ within the measurement volume is discretized into voxel elements. The object projections onto the camera sensors are given in form of digital images, where the intensity is represented on the pixel array with intensity $I\left(x_{i}, y_{i}\right)$. For the sake of simplicity, the digital resolution of the physical space by voxels is assumed to be approximately the same as that of the images. Therefore, the voxel size is comparable to that of the pixel image. The projection of the light intensity scattered by particles on the camera sensors can be written in discrete form as follows:

$$
\sum_{j=1}^{N} w_{i j} E_{j}=I_{i},
$$

where $N$ represents the number of voxels affecting the intensity value of the $i$ th pixel and $i=1,2, \ldots, M$, where $M$ is the total number of pixels. The weighting coefficient $w_{i j}$ describes the contribution of the $j$ th voxel to the $i$ th pixel. The calculation of the weighting coefficient is simplified when considering spherical voxels with volume equivalent to that of the cubic voxel. More details are given in Elsinga (2006a). The line-ofsight may also be modeled as cylindrical with a cross-sectional area of the pixel. The intensity field at the first iteration $E^{1}$ is estimated from the initial guess $E^{0}$ through the update equation:

$$
E_{j}^{1}=E_{j}^{0}\left(\frac{I_{i}}{\sum_{j \in N_{i}} w_{i,} E_{j}^{0}}\right)^{\mu w_{i j}},
$$

where $\mu$ is a relaxation parameter, typically set to a value of 1 or smaller. The initial guess is commonly chosen as a uniform small value.

\subsection{Accuracy of the reconstruction}

The accuracy of the reconstruction can be quantified by the quality factor $Q$ (Elsinga et al 2006b), computed as the normalized cross-correlation coefficient between the actual solution (usually only available for computer-simulated problem) and the reconstructed intensity field:

$$
Q=\frac{\sum E_{\text {actual }} \cdot E_{\text {rec }}}{\sqrt{\sum E_{\text {actual }}^{2} \cdot \sum E_{\text {rec }}^{2}}} .
$$

For iterative MART, the quality factor $Q$ and the measured velocity field by cross-correlation analysis, typically do not change appreciably after five iterations (Elsinga et al 2006a), which is commonly chosen as the stop criterion also in view of the large computational cost of MART. The reconstruction accuracy is affected by different types of error, which may be classified into three categories:
(I) the representation of the intensity field into voxels introduces discretization errors, which become important when particle images of small diameter (below 3 voxels) are reconstructed;

(II) the viewing geometry and mostly the solid angle subtended by the tomographic imaging system may lead, if too small, to elongated particles along the depth, for a typical angle between cameras ranging between $40^{\circ}$ and $60^{\circ}$ (Wieneke and Taylor 2006). This condition slightly increases the measurement uncertainty for the velocity component along the depth.

(III) ghost particles (Maas et al 1993) are formed at the intersection of the lines of sight where particles are simultaneously present. For a high seeding density, this effect is also reported to be the dominant source of error in tomographic PIV measurements (Elsinga 2008).

The present study deals with the third type of problem.

\subsection{Ghost particles}

Ghost particles arise from the non-uniqueness of solution to the under-determined algebraic problem defined by equation (1). They consist of spurious intensity peaks appearing in the reconstructed intensity field together with the actual particles. The presence of ghost particles introduces also artifacts in the cross-correlation analysis and ultimately affects the measurement and therefore the velocity fields (Elsinga et al 2009). Moreover, the energy captured by the ghost particles is subtracted from that of the actual particles, decreasing the accuracy of the latter. It was assessed by Elsinga (2008) that a value of $Q$ of 0.75 or higher yields a reconstruction quality that enables a reliable analysis of the tracers motion by cross-correlation. Because $Q$ strongly depends upon the imaged seeding density, the seeding concentration of Tomo-PIV experiments must be limited to levels typically not exceeding 0.05 particles per pixel (50000 particles/megapixel for a four-camera system) according to the simulations of Elsinga et al (2006b).

The number of ghost particles produced by MART can be comparable and often larger than the number of actual particles, and it depends mainly on three experimental parameters, namely the number of simultaneous views, the seeding density and the thickness of the illuminated volume (Maas et al 1993). Also, according to Elsinga et al (2006a), the ratio of actual to ghost particles which represents a possible indicator of the signal-to-noise ratio can be modeled as follows:

$$
\frac{N_{p}}{N_{g}}=\frac{1}{p p p^{N_{C}-1} \cdot A_{p}^{N_{C}} \cdot l_{z}},
$$

where $N_{C}$ represents the number of cameras, $A_{p}=\frac{\pi d_{\tau}^{* 2}}{4}$ is the particle image area in pixels and $l_{z}$ is the thickness of volume along the depth direction. The above relation would require a correction to account for oblique lines of sight, whereby imaging with a larger solid angle produces an increase of particle image density on the imagers. It is also useful to introduce the source density $N_{S}$ (Keane and Adrian 1990), which takes into account both the number of particle images per unit sensor area and the diameter of individual particle 

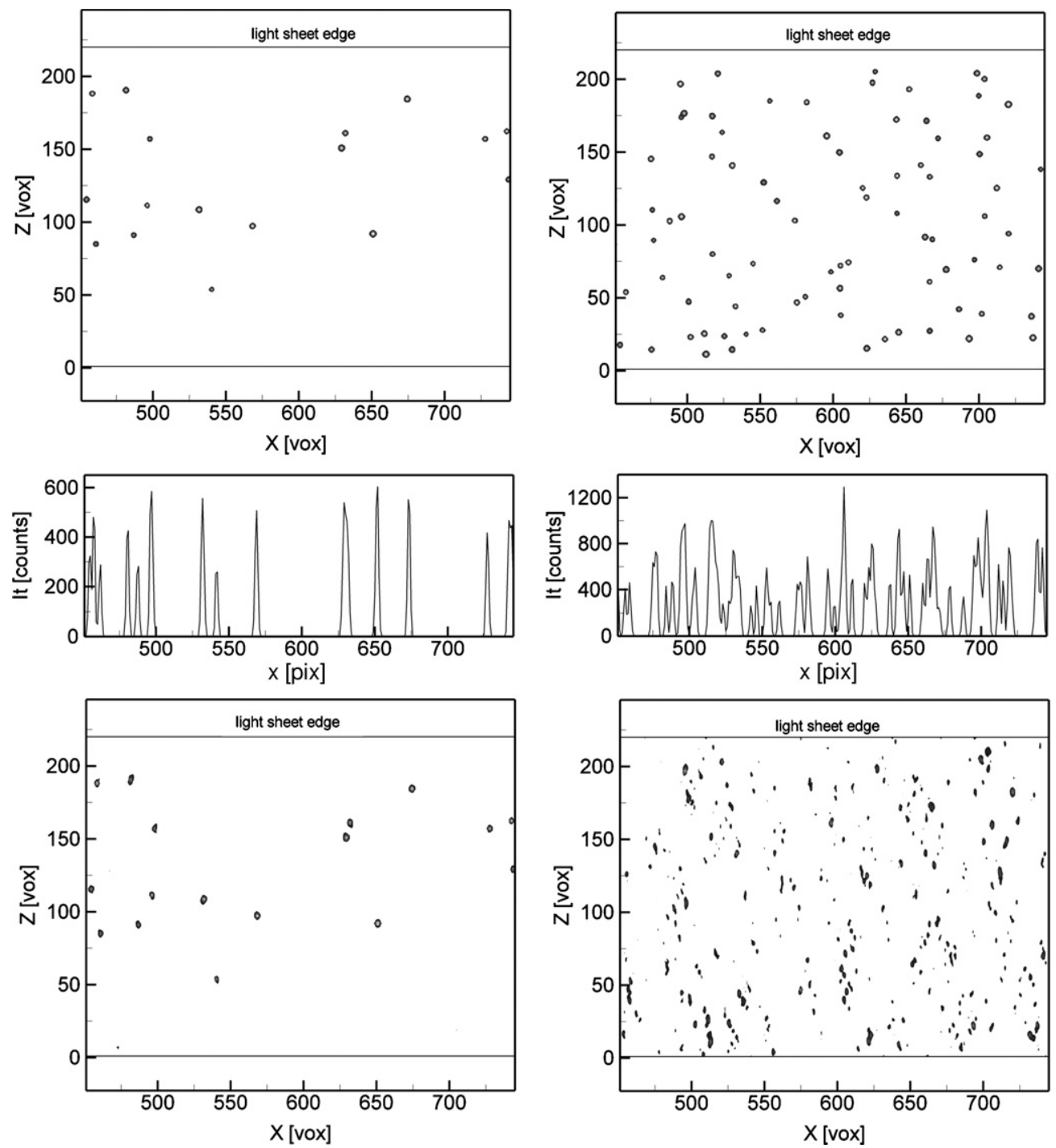

Figure 1. Numerically simulated 2D intensity field (top) and reconstructed field by MART (bottom). $N_{S}=0.15$ (left) and $N_{S}=0.9$ (right). Intensity distribution on the $1 \mathrm{D}$ camera sensor at $0^{\circ}$ (middle).

images. Since $N_{S}=p p p \cdot \frac{\pi d_{\tau}^{* 2}}{4}$, the ratio between the number of actual particles and of produced ghosts reads

$$
\frac{N_{p}}{N_{g}}=\frac{1}{N_{S}^{N_{C}-1} \cdot A_{p} \cdot l_{z}} .
$$

In this form, the dependence upon the number of simultaneous views is only present in the exponent of the source density. Instead the effect of the particle image diameter and reconstruction depth is independent of $N_{C}$. Ghost particles form at the intersection of the lines of sight (LOS) where pixel values attain a local maximum. This leads to ambiguity in the reconstruction as illustrated in figure 1. Therefore, the object reconstruction produced by the MART algorithm can converge to an incorrect solution. Also the depth of the measurement domain affects the formation of ghosts in that the LOS intersection through the object determines the probability of overlapping of particle images. In this respect, the role played by the viewing angle is twofold: increasing the viewing angle, the length of the intercepted segment along the LOS increases, which causes an undesired increase of the seeding density on the imager; on the other hand, a small viewing angle produces reconstructed particles that are elongated along the depth. The reconstruction accuracy attains a maximum around a viewing angle of $30^{\circ}$ according to Elsinga (2008), which is therefore retained for the simulations carried out in the present study.

To illustrate the dependence of the reconstruction accuracy upon the source density, figure 1 shows the results of a two 2D simulation using four viewing directions, with a total aperture angle of $60^{\circ}$ at values of the seeding density corresponding to that commonly adopted in experiments $\left(p p p=0.05, N_{S}=0.15\right)$ and far beyond it $\left(p p p=0.3, N_{S}=\right.$ $0.9)$. The particle images diameter is 3 pixels and the results are shown for five MART iterations. The present results can be transposed to the $3 \mathrm{D}$ case adopting the same value of $N_{S}$ or dividing the $p p p$ by the particle image diameter. As a result, the equivalent particle image density for the $3 \mathrm{D}$ case would be 0.0167 and $0.1 \mathrm{ppp}$ for the low and high concentration cases, respectively. The effect of seeding density is clearly visible, whereby the reconstruction at low particle concentration does 
not show any significant artifact because the probability of particles' interaction along the same lines of sight is rather low. Instead at high $N_{S}$ a significant degradation of the actual particles occurs with intensity transferred from the latter to the ghost particles field. The value of the quality factor evaluated for the two reconstructed fields is $Q=0.95$ and $Q=0.5$, respectively. The second case corresponds to a situation where no reliable Tomo-PIV experiment can be performed. Much attention is therefore devoted to the proper selection of the seeding concentration so as to optimize tomographic PIV experiments and reach the maximum allowed seeding density in order to increase the spatial resolution without compromising the reconstruction accuracy.

The technique proposed in the present study aims at enhancing the reconstruction accuracy at levels of the seeding concentration that are beyond (typically from two to three times) the current limits of Tomo-PIV experiments.

\section{Theoretical model for MTE}

The mathematical problem of tomographic reconstruction by algebraic methods from a small number of views is underdetermined and several solutions exist in principle for a given set of images. It has been shown, however, that for PIV recordings at relatively low density, the MART technique returns a reliable and accurate estimate of the actual intensity field $(Q>0.9)$. Moreover, it was shown recently by Elsinga et al (2009) that many ghost particles do not produce a coherent pattern in the correlation map, which makes the crosscorrelation analysis of tomograms significantly less affected by ghost particles than methods based on individual particle detection and tracking.

On the other hand, when the seeding concentration increases, the solution of the MART reconstruction becomes less accurate, as shown in figure 1, and also cross-correlation analysis may fail in determining the actual particle motion.

A crucial point that is not covered in the existing literature, which is dealt with in the present study, is the dependence of the MART solution upon the initial guess. This is associated with the undetermined nature of the solution of the algebraic problem posed by the reconstruction.

\subsection{Geometrical analogy for MART solutions representation}

Iterative projection method convergence does lead to a solution which minimizes the distance from the initial guess in the space of the solutions. Therefore, different initial intensity fields can result in different reconstructed objects. This behavior becomes clear when the exact intensity field is considered as initial guess; in this case, the iterative reconstruction algorithm returns the exact solution after the first iteration, without altering the initial guess.

The main reason why probably this aspect has not been dealt with is that it is difficult to build a priori information on the particles' random distribution. The most common approach to the problem is to deny any initial information and assume a uniform (non-zero) intensity distribution to start the reconstruction algorithm (e.g. by MLOS or MART).

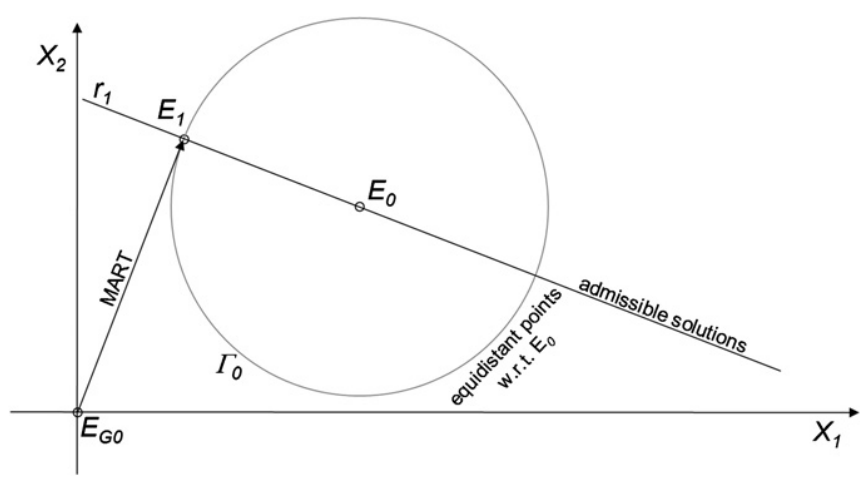

Figure 2. Geometrical interpretation of the space of solutions for a two-voxel/one-equation system. Initial value for MART is close to the origin. The circle indicates all solutions that are equidistant from the exact solution.

The problem requires a representation in hyperspace; however, for the sake of clarity the case of an object composed of only 2 voxels and with a single projection equation is illustrated in figure 2. The solution is represented as a point in $2 \mathrm{D}$ space. The single equation admits solutions belonging to a straight line $r_{1}$. If the initial condition is chosen as a point in the neighborhood of the origin $E_{G 0}$ (as commonly done for MART object initialization), the application of the MART update equation will project the result onto $r_{1}$, returning a solution $E_{1}$ at a distance $\left|E_{1} E_{0}\right|$ from the exact solution.

For usual problems, the latter distance is known to depend mostly upon the number of simultaneous views $N_{C}$ and the particle image density $N_{S}$. Therefore, for a given value of the latter parameters, any MART reconstruction yields a solution that may lay on a circle centered on $E_{0}$.

The problem generalization to Tomo-PIV operating conditions requires an $\mathrm{N}$-dimensional space to represent all possible solutions ( $N$ independent voxels). Projection equations available for the $M$ pixels (with $M \ll N$ ) are provided by the imaging system. Each equation defines a hyper-plane, and the combination of the $M$ hyper-planes defines a sub-space where the admissible solutions exist. The mentioned sub-space depends upon the given initial condition. In case the initial condition coincides with the exact solution, the sub-space only contains one point, the exact solution. Because the problem is strongly under-determined, the option of introducing a few more additional projection equations will not yield a unique solution (it may be wrongly suggested by the graphical representation in figure 2 based on a 2D space: adding a second non-parallel line to $r_{1}$, their intersection appears uniquely determined). This is because the number of additional projections must be orders of magnitude beyond that of the available projections. Instead, the current discussion focuses on the fact that if a better initial condition were available, for instance if the point $E_{G 0}$ is slightly moved to the right, then the distance between the MART solution and the exact value will decrease.

\subsection{Iterative first guess evaluation}

Consider now that a hypothetical second solution of the same problem is available, for instance obtained from a second view 


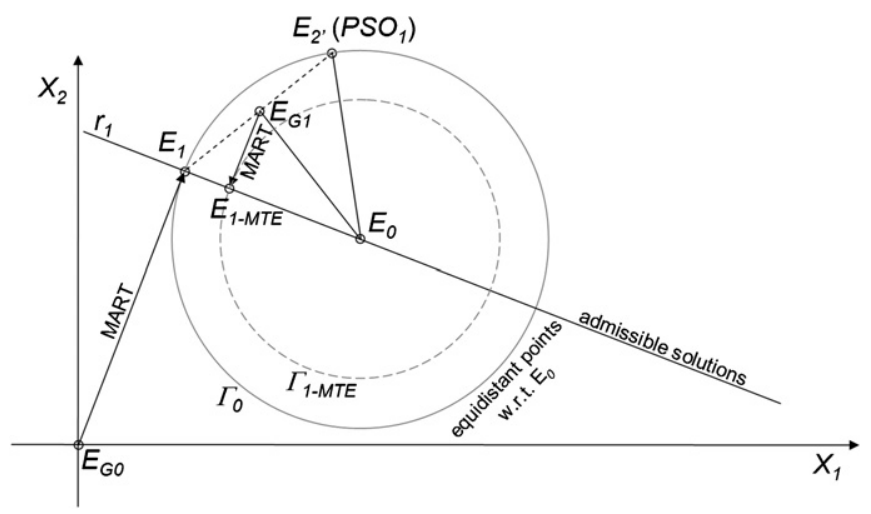

Figure 3. Utilization of two equidistant (non-coinciding) solutions to obtain an enhanced first guess for MART leading to a result closer to the exact solution.

of the same set of particles at a subsequent time instant. We denote this solution as $E_{2^{\prime}}$ to indicate that it follows $E_{1}$ in time (figure 3 ). The prime symbol indicates that this view has been reported at the same time instant as $E_{1}$ by knowledge of the particle motion field and as such is denoted as the pseudosimultaneous object (PSO, see details in the next section). In this case $E_{2^{\prime}}$ is expected to lay on the circle of equidistant solutions obtained starting from $E_{G 0}$ because it is derived from the same number of views $N_{C}$ and with the same image source density $N_{S}$. However, if the particle motion involves velocity differences along the depth, the point $E_{2^{\prime}}$ will be at a different point on the circle $\Gamma_{0}$, indicating that the ghost particles will occur with a different pattern. A linear combination of $E_{1}$ and $E_{2^{\prime}}$ already yields a result $E_{G 1}$ at a lower distance from $E_{0}$. When this is used as a first guess for MART, the result will project onto $r_{1}$ reaching a position that is further approaching the exact solution $E_{0}$.

Also for this case, the iterative application of this technique should not be expected to converge to the exact solution, because the problem remains largely underdetermined. The important point in this approach is that different views (partly independent) of the same object are utilized for the reconstruction of an individual object. The combination of the two reconstructions returns the enhanced guess object, whose projection is expected to be closer to the exact solution when using the MART update equation. Key to this approach is the estimate of the particles motion in order to reduce non-simultaneous exposures to the same pattern. The method therefore enhances the MART reconstruction by tracking the motion of the particles (MTE).

The result indicated as $E_{1-\text { MTE }}$ must be combined with an enhanced reconstruction from the second exposure to form the objects pair for cross-correlation analysis.

\section{MTE algorithm}

The 3D particle pattern undergoes a transformation between the two (or more) exposures, which requires the particle motion field to be known or estimated by some means in order to deform the object intensity to a single time instant. Because a priori information on the motion field is not available, the method can only be implemented with an iterative algorithm.
The main objective of the MTE is that of iteratively building a first guess for the $3 \mathrm{D}$ intensity field $E_{G}$ to be used as input for the MART algorithm. This guess is obtained combining the information from the first and second (or a multiple set) exposures as previously illustrated in figure 3 making use of the MART reconstruction, cross-correlation analysis and object deformation technique similar to that used in the interrogation of the objects. The MTE method can be schematically described by five main steps:

(1) MART reconstruction of individual recordings, returning $E(\boldsymbol{R}, t)$ and $E(\boldsymbol{R}, t+\Delta t)$, shortly $E_{n}$ and $E_{n+1}$;

(2) cross-correlation analysis of the pair of objects $E_{n} \otimes E_{n+1}$, which yields the particles velocity $\boldsymbol{V}(\boldsymbol{R}, t+\Delta t / 2)$, shortly $V_{n}$;

(3) evaluation of the pseudo-simultaneous object (PSO) by deforming one particle field according to the estimated motion field. $E_{n+1^{\prime}}=E(\boldsymbol{R}+\boldsymbol{V} \cdot \Delta t, t+\Delta t)$ and $E_{n^{\prime}}=$ $E(\boldsymbol{R}-\boldsymbol{V} \cdot \Delta t, t)$

(4) linear combination of the two objects: $E_{G n}=\frac{1}{2}\left(E_{n}+\right.$ $\left.E_{n+1^{\prime}}\right)$ and $E_{G n+1}=\frac{1}{2}\left(E_{n+1}+E_{n^{\prime}}\right)$

(5) input the result as first guess for MART reconstruction: $E^{0}=E_{G n}$.

In the above points, the vector $\boldsymbol{R}=(X, Y, Z)$ indicates the coordinates in the object space. After step 5, the sequence from 1 to 4 can be repeated several times until the result exhibits convergence.

The first two steps are straightforward and do not require specific discussion. The deformation of one object (e.g. from the exposure at time $t+\Delta t$ ) to obtain the intensity field that would be expected at time $t$ is made by the deformation technique used for the 3D iterative interrogation (discussed for instance in Scarano and Poelma (2009)). The following sections discuss the way the information from different exposures is combined, the initialization of the MART iterative calculation and the overall iterative structure of the MTE algorithm.

\subsection{Suppression of incoherent (ghost) intensity}

The operating principle of the MTE technique is visually explained by showing how the structure of the ghost intensity field evolves at subsequent time instants. A ghost particle is returned by the MART reconstruction at the intersection points of different lines of sight corresponding to pixels characterized by non-zero intensity. Thus, the ghost particle position depends on the relative position of the actual particles and the lines of sight of the imaging system. This means that, while the actual tracers move from one exposure to another according to the flow motion, the ghosts generally disappear or move along different directions since the relative position of the actual particles and the viewing cameras can change, which depends on the particle tracers' displacement field. This aspect is discussed in more detail in Elsinga et al (2009).

Figure 4 (left) illustrates the case of two particles $A$ and $B$ forming two pairs of ghosts $G_{1}$ and $G_{2}$ at the first and second exposure, respectively. For the given displacement field, the two sets of ghost particles do not occur at the same location. 

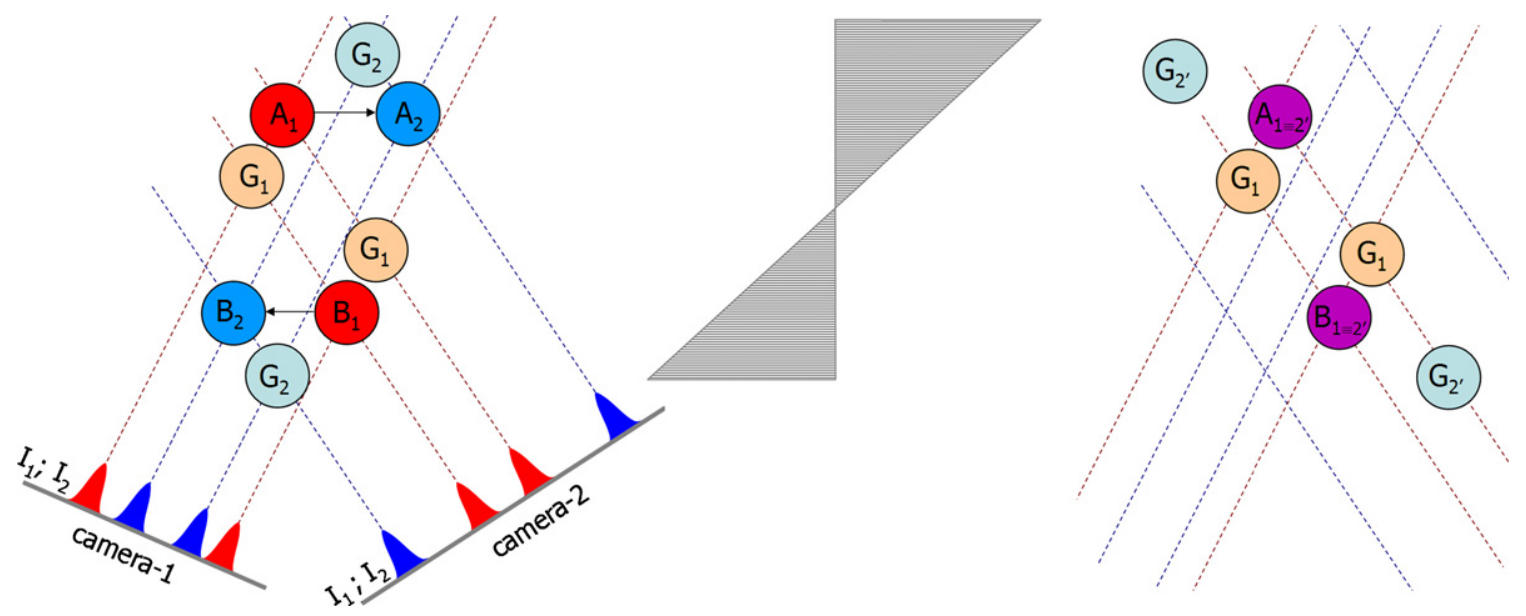

Figure 4. Left: two particles $A$ and $B$ imaged from two directions exposed twice (first exposure field ' 1 ' in red and second exposure ' 2 ' in blue). Particles move according to a hypothetical displacement field (gray pattern on the left). The ghost particles produced by MART represented in lighter colors. Right: superimposed actual particles (purple) and ghost field after that the second exposure field is tracked back to the first exposure time considering the displacement field known.

The necessary condition for this to occur is that the difference between the displacements of the particle tracers from which the ghosts are produced is larger than the diameter of the reconstructed particle. If an estimate of the displacement field is available, the intensity field reconstructed at the second time instant can be deformed according to the displacement field into that at the first time instant returning the PSO. The positions of the particles $A^{\prime}{ }_{2}$ and $B_{2}^{\prime}{ }_{2}$ will in this case coincide with those of $A_{1}$ and $B_{1}$, whereas $G_{1}$ and $G_{2}$ do not necessarily interfere.

If the fields 1 and $2^{\prime}$ are considered, two independent observations and reconstruction of the same particle field undergoing a transformation due to the particles motion are used to enhance the reconstructed particle field at either of the two time instants. For instance, multiplying the intensity will retain the (coherent) intensity associated with the actual particles and suppress most of the (incoherent) intensity associated with the ghost particles. However, any error in the estimate of the displacement field will also introduce a cancellation of the coherent intensity field. Instead, averaging the two intensity fields will not eliminate coherent intensity even in the case of local erroneous velocity information. This aspect is further detailed in the remainder. As a drawback, the incoherent intensity is not suppressed as rapidly as in the case of multiplication, but only halved after every iteration. The reconstructed intensity field can be decomposed into the coherent $E_{C}$ and incoherent $E_{I}$ parts, respectively:

$$
E_{\mathrm{MART}}=E_{C}+E_{I} \text {. }
$$

The term $E_{C}$, although associated with the actual particles, does not correspond exactly to the actual intensity field $E_{0}$ (accounting for all the energy in the reconstructed volume) because part of such energy is captured by the ghost particles.

The above decomposition is valid at each of the time instants when a reconstruction is made. If the second exposure is deformed toward the first time instant by knowledge of the displacement field ( $\left.2^{\prime}\right)$, one obtains that $E_{C}$ mostly coincides from the two time instants, instead $E_{I}$ will not correspond.
The deformed particle field is called the pseudo-simultaneous object (PSO), as introduced at step 3 of the MTE sequence. In more detail

$$
\begin{aligned}
& E_{2^{\prime}}(X, Y, Z) \equiv \mathrm{PSO}_{1}=E_{2}\left(X+U_{\text {pred }} \cdot \Delta t, Y\right. \\
& \left.\quad+V_{\text {pred }} \cdot \Delta t, Z+W_{\text {pred }} \cdot \Delta t\right) .
\end{aligned}
$$

Here, the subscript pred indicates a velocity predictor from cross-correlation. In the hypothesis that velocity predictor is accurate within a particle image diameter, the average between the two fields can be written as

$$
E_{G} \approx E_{C}+\frac{1}{2}\left(E_{I, 1}+E_{I, 2^{\prime}}\right)
$$

which indicates that the above procedure would keep unaltered the coherent part of the signal (actual particles) and decrease the peak intensity of the ghost particles that do not travel according to the actual particles velocity. An initial estimate of the particle motion is to be produced by cross-correlation analysis between the two reconstructed objects. As a consequence, the MTE method will effectively reduce the ghost intensity field only if the estimate of the particle motion is at least reliable. A first approach could be to consider as a tomographic experiment design rule that proposed by Elsinga et al (2006b) in a less conservative way and at a lowered spatial resolution (e.g. increased interrogation block size).

\subsection{MART first guess and iterative evaluation}

The use of the intensity field $E_{G}$ to initialize the MART computation is demonstrated to further improve the accuracy of the reconstructed object as graphically described in figure 3 , because the ghost intensity field $G_{2}^{\prime}$ is rapidly eliminated by the MART algorithm where these particles do not fall at positions where actual intensity is present on the images. Recalling figure 4 (right), the light blue particles do not correspond to the intensity field recorded from both cameras. Equation (2) is therefore evaluated using $E_{G}$ instead of a uniform intensity field. The evaluation may be iterated until convergence is attained to a good degree. This procedure is performed for 


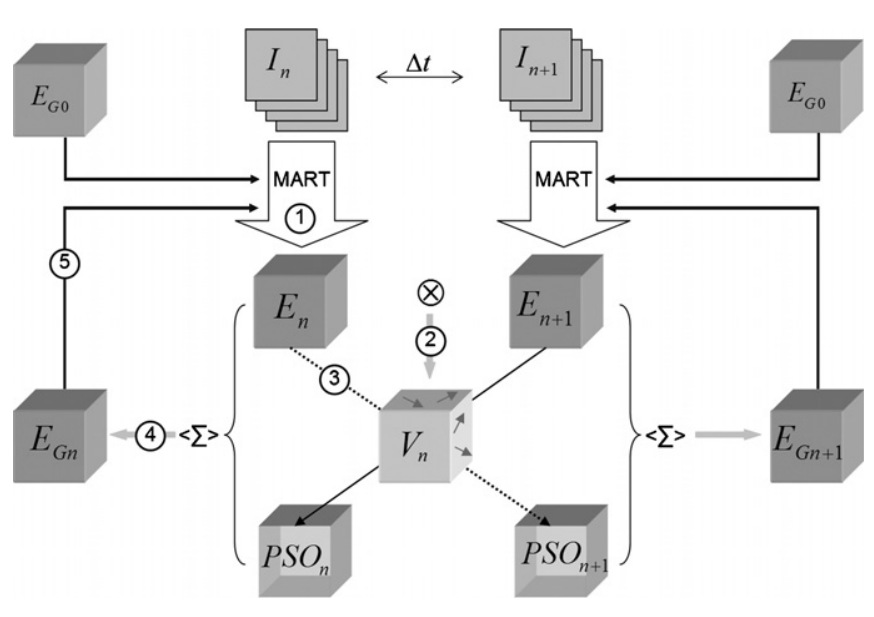

Figure 5. Flow chart of the MTE-MART technique for double-frame recordings. (1) MART reconstruction of individual recordings; (2) cross-correlation analysis of the object pair;

(3) evaluation of PSO by deformation of the objects according to the motion field; (4) averaging the reconstructed and pseudo-simultaneous objects; (5) input result as first guess of the next MART reconstruction.

the reconstructed objects from both exposures, yielding an enhanced intensity field at both time instants $E_{1}^{\mathrm{MTE}-1}$ and $E_{2}^{\mathrm{MTE}-1}$, where the subscript indicates the order in the exposure and the superscript the number of times that MTE is repeated.

One MTE iteration step consists of the operations listed above from points 1 to 5: the MART reconstruction of two tomographic recordings $E_{n}$ and $E_{n+1}$, the cross-correlation between the reconstructed objects, the deformation of the objects to obtain the pseudo-simultaneous fields $\left(\mathrm{POS}_{n}\right.$ and $\mathrm{POS}_{n+1}$ ) and the evaluation of the intensity field to be used as first guess $E_{G n}$ for the subsequent application of the MART algorithm. The enhanced intensity fields are again interrogated by cross-correlation analysis yielding an updated displacement field, which can be used in turn to further enhance the two reconstructed and enhanced objects.

Successive iterations of the method are expected to progressively improve the results because the initial guess for the velocity field may be inaccurate as a result of the large number of ghost particles. Therefore, at following iterations, the ghost intensity is progressively reduced increasing the accuracy of the displacement field either for a final output or for a successive enhancement. The number of enhancement steps, referred to as $N_{E}$, is therefore a crucial parameter expected to affect the result of the MTE technique. Other important factors are the structure of the velocity field, the diameter of the tracer particle images and the viewing angles of the imaging system. Moreover, the MTE-MART technique can also be applied in multiple-frame tomographic experiments.

A synthetic description of the algorithm is given in the form of a flow-chart (figure 5) for the double-frame operating mode. In the case of continuous PIV recordings, as typically obtained by high-repetition rate PIV systems, the availability of time-resolved data allows us to build the pseudo-simultaneous object for the $n$th recording using one or more exposures acquired before and after the considered time instant. The number of recordings used to create the PSO will be denoted by $N_{O}$; for the double-frame mode $N_{O}$ is equal to 2 .

\subsection{Numerical evaluation}

The effect of the MTE method on the reconstruction is simulated with a 2D numerical model and the results are shown in figure 6. The displacement field between the exposures was chosen as a shear along the depth of the domain similar to what is described in figure 4; the maximum displacement between the exposures is 5 voxels. The tomographic recordings are characterized by a high seeding density $\left(N_{S}=0.9\right.$, ppp $=$ 0.3 ), and the imaging system is composed of four cameras. For the sake of clarity, the results are shown in a small region of the complete reconstruction volume $(1000 \times 200$ voxels). The actual particle field distribution is indicated by circles at locations where particles are placed. The MART reconstruction in these conditions reproduces most of the particles but at a reduced intensity and with distorted shape. The corresponding quality factor $Q$ is 0.55 ; a value well below the criterion proposed by Elsinga et al (2006a) for a reliable correlation $(Q>0.75)$. Moreover, a significant amount of ghost particles with intensity comparable to that of the actual particles is returned. Averaging the reconstructed object and its PSO from a pair of exposures (figure 6, top-right) returns some ghost intensity reduction and improves the precision on the position of the actual particles $(Q=0.6)$. Performing a MART computation that takes as initial guess the result on the top-right yields a result shown in the bottom-left picture. An improvement comparable to the previous step is observed $(Q=0.65)$. The iterative application of MTE approaches an asymptote after approximately 25 steps; here the result after 10 steps is shown $(Q \approx 0.85)$, a value above the criterion for accurate cross-correlation analysis.

In conclusion, the improvement obtained with the MTE technique can be seen as the result of two contributions:

(1) the ghost particles intensity is progressively reduced;

(2) consequently, the intensity is redistributed among the actual particles, which are reconstructed more accurately.

Regarding the cross-correlation procedure, the second aspect is particularly significant; since the MTE method gives the best results when the ghost intensity displacement is poorly correlated to the flow motion (Elsinga et al 2009), one can expect that this contribution will not affect the crosscorrelation signal significantly, but only reduce the effect of the ghost intensity field. As will be shown in section 5, also the velocity field resulting from correlation of enhanced objects is more accurate.

\section{Numerical performance assessment}

The detailed effect of the main MTE parameters variation on the measurement accuracy is investigated by computersimulated tomographic images of a known flow field. A two-dimensional object is projected onto one-dimensional virtual sensors, similar to the approach followed by other studies (Elsinga et al 2006a, Atkinson et al 2008, Worth 

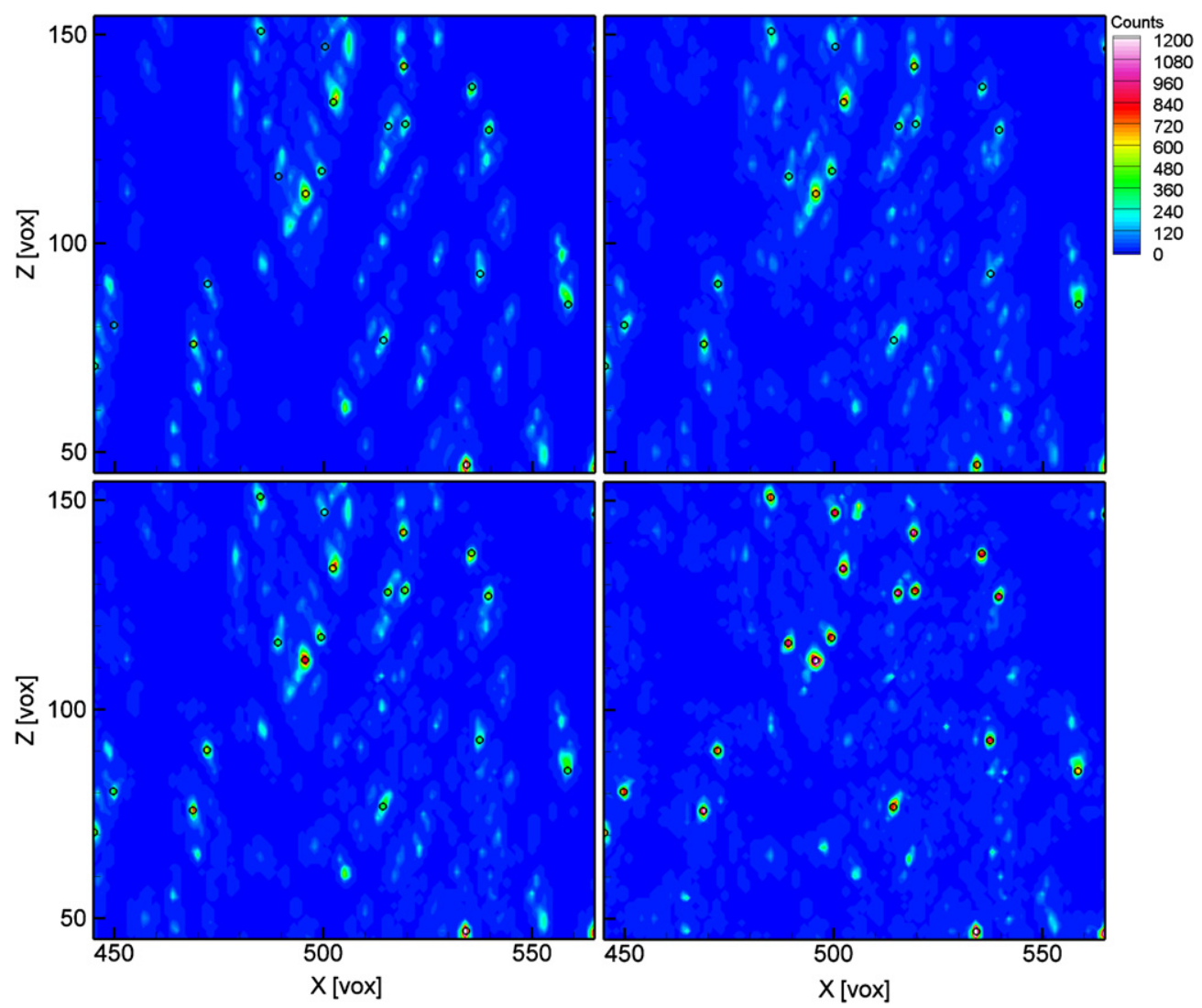

Figure 6. Top-left: MART reconstructed intensity field for one exposure. Top-right: intensity field used as initial guess for the first MTE step. Bottom-left: reconstructed field after one MART iteration of the first enhancement step. Bottom-right: reconstructed fields after ten MTE-MART steps. The position of the actual particles is indicated by solid black circles.

and Nickels 2008). Particle images are generated within objects of $1000 \times 200$ voxels at random locations by the Monte Carlo technique. The diameter of the particle images expressed in pixel units $d_{\tau}^{*}$ is set to 3 , a representative value for Tomo-PIV experiments reported in the literature. The particle tracer concentration in the object is varied in such a way as to obtain a particle image density ppp on the imager ranging between 0.05 and $0.3\left(N_{S} \in[0.15,0.9]\right)$. The particle displacement field is a sinusoidal shearing motion of 200 voxels wavelength and 3 voxels amplitude, representative of shear layer flows measured with the velocity gradient aligned with the depth direction. The object is imaged by onedimensional array sensors of 1000 pixels size, with uniform angular displacement $(\vartheta=\{-30,-10,+10,+30\}$ degrees $)$. The particle image density and the number of cameras are reported to be the most important parameters governing the tomographic reconstruction process. The number of viewing cameras $N_{C}$ is varied from four to eight. To date, most TomoPIV experiments are performed with values of $p p p \in[0.02$, $0.1]$ and $d_{\tau}^{*}=2-4$. The range investigated in the present simulation may initially appear unrealistic because it extends far beyond the currently practiced values. However, it will be discussed later that the measurement range extension, making use of MTE, justifies the present choice of the simulation range.

The parameters governing the MTE method are the number of iterative enhancement steps $N_{E}$ and the number of objects involved in the MART enhancement $N_{O}$. The discussion initially considers the case $N_{O}=2$ (doubleframe images). Subsequently, the simulation is performed for the case of more than two reconstructed objects (timeresolved imaging) obtained from subsequent exposures, which correspond to the case of time-resolved measurements performed in continuous single-frame mode $\left(N_{O}=\{3,5\right.$, 7\}). Two evaluation criteria are used: the reconstruction quality factor $Q$ and the standard deviation of the velocity measurement error $\sigma$. The computational efficiency of the MTE technique is not discussed in the present work; therefore, the computation time is not taken into account here as a performance parameter.

\subsection{Double-frame recordings}

The quantitative evaluation of the effects shown in figure 6 is given by the occurrence histogram of the particle peak intensity $I_{p}$ illustrated in figure 7 . Distinction is made between the peak intensity of actual particles (black) and that of ghost particles (grey). From the MART reconstruction, the reconstructed actual particle peak intensity occurrence attains a maximum at 100 counts and extends till about 500 counts. Considering that the exact value of the generated particle peak intensity is centered on 1000 counts, the MART reconstruction in these conditions does not retrieve more than $10 \%$ of it. The distribution of the ghost particles exhibits 

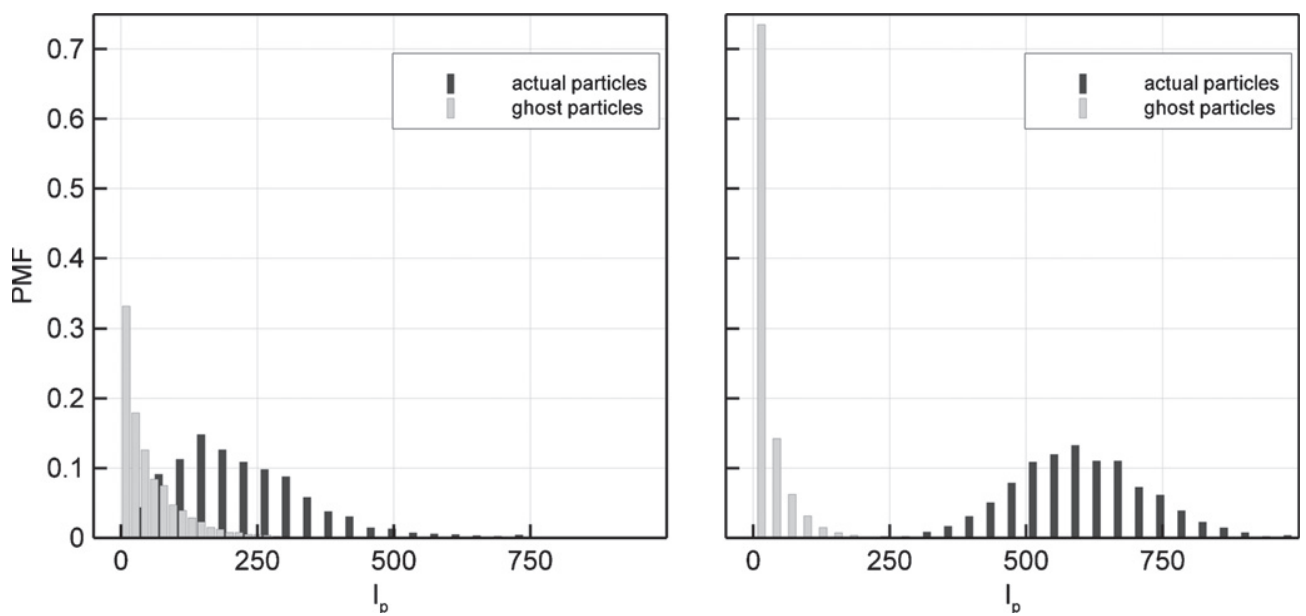

Figure 7. PMF of particle peak intensity for actual and ghost particles in the reconstructed object. Left: MART reconstruction $\left(N_{S}=0.3\right.$, $\left.N_{C}=4\right)$. Right: MTE-MART after ten enhancement steps.
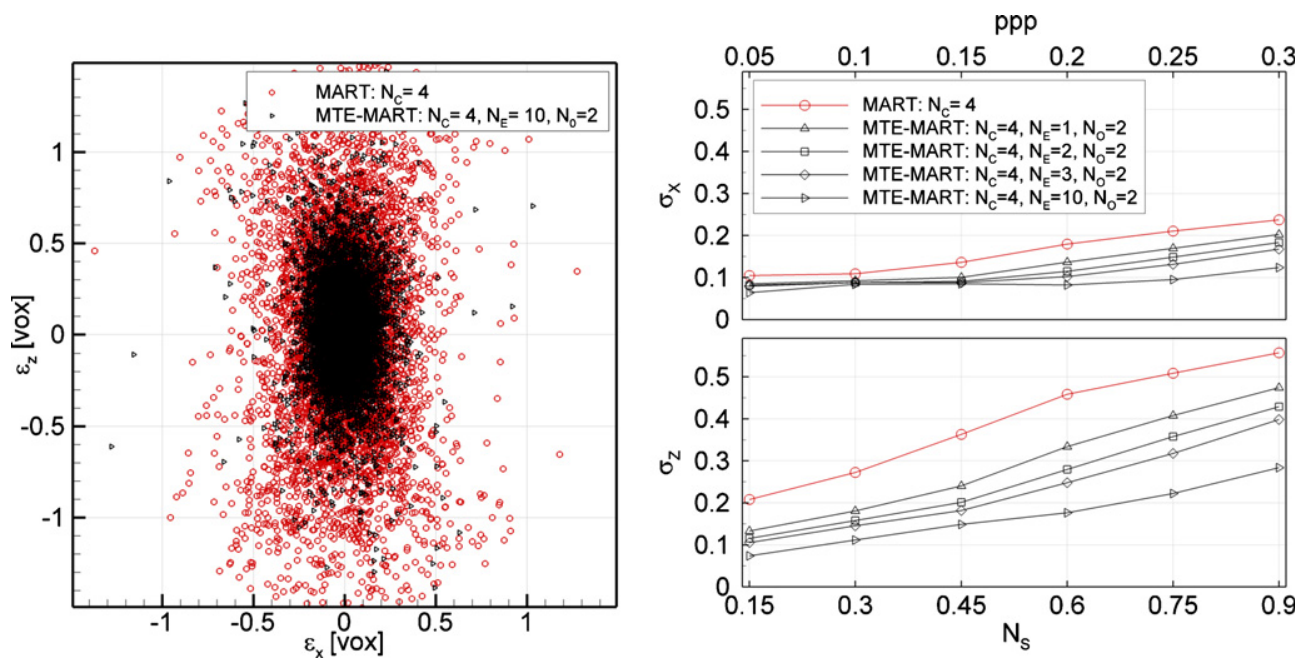

Figure 8. Left: 2D error dispersion plot of the difference between the actual and the reconstructed particle peak positions $\left(N_{S}=0.9\right)$. Right: standard deviation of the error in the peak position as a function of $N_{S}(p p p)$ varying the number of enhancement steps $N_{E}$.

lower values in agreement with previous findings from Elsinga (2008). However, under these conditions, the distributions of actual and ghost particle intensity overlap to a large extent, which decreases the signal-to-noise ratio of the reconstructed object. The application of MTE by ten iterative steps (figure 7 , right) strongly alters the situation. First, the peak intensity distribution of the actual particles reconstruction is raised by a factor 6 with a maximum occurrence attaining 600 counts. Secondly, the ghost intensity is slightly reduced with a significantly higher number of low-intensity ghost particles. In this case, MTE allows us to completely separate the histograms of actual particles and ghosts. Also the precision of the individual particles reconstruction and their peak location appears to be improved. The precision error of the reconstructed particle (Gaussian) centroid location $\varepsilon$ is represented with a $2 \mathrm{D}$ dispersion plot (figure 8 , left), where $\varepsilon_{Z}=Z_{R}-Z_{0}$ is the difference between the measured position of the reconstructed particle $Z_{R}$ with respect to the exact position $Z_{0}$; the same applies for the position along $X$. It can be observed that the precision error in the location of the particle centroid is considerably reduced when the MTE technique is applied, which also follows from the observations made in figure 6. Not surprisingly, the error along the $Z$ coordinate direction has a higher dispersion than that along the $X$-direction (in-plane). The dependence of the particle centroid position error $\varepsilon$ upon the source density $N_{S}$ is given from the ensemble standard deviation $\sigma$. The diagrams indicate that $\sigma_{X}=0.1-0.2$ voxels and about twice as much for $\sigma_{Z}$ when MART alone is applied. Adopting MTE reduces these errors by factors 2 to 3 depending on the value of $N_{S}$. This also indicates that MTE-MART could be a well-suited technique to improve the measurement precision for experiments that require individual particle tracking (PTV) in 3D (e.g. Schröder et al 2009).

The reconstruction accuracy is quantified by the quality factor $Q$ and depends mostly upon the number of viewing cameras $N_{C}$ and the source density $N_{S}$ (Elsinga et al 2006a). The comparison of the accuracy returned by MART and that obtained by MTE in the present study is also shown for different values of the number of enhancement steps $N_{E}$ 

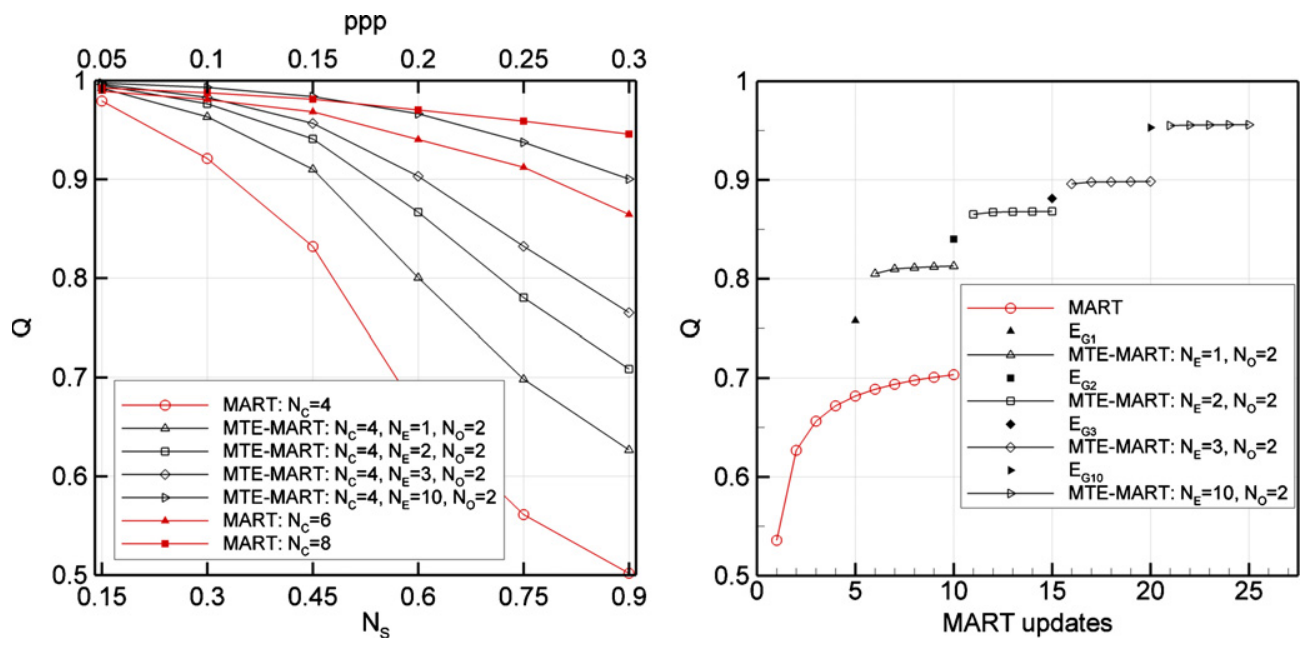

Figure 9. Left: reconstruction quality factor obtained by MART and MTE-MART (double-frame recordings) as a function of particle image density $N_{S}(p p p)$. Curves are given for varying number of enhancement steps. Right: effect of iterative MART analysis and initial guess enhancement $\left(N_{S}=0.6, N_{C}=4\right)$.

(figure 9, left). The dependence of $Q$ upon the seeding density for MART is in agreement with previous studies. The criterion stated by Elsinga $(Q>0.75)$ may be taken as an upper limit for the seeding density in Tomo-PIV experiments, which is currently around $N_{S}=0.5$ for a system with four cameras.

Results from MTE-MART show that it is possible to increase the accuracy of the reconstruction and in turn extend the limit for the maximum seeding density: $Q$ approaches 0.9 even for a seeding density corresponding to approximately $N_{S}=1$ if ten MTE steps are applied. Moreover, the effect of the enhancement technique is comparable to that of increasing the number of cameras; the asymptotic behavior of the MTE-MART reconstruction compares well with the MART reconstruction when doubling the number of simultaneous views.

The relative importance of the MART iterations and the guess intensity field to the reconstruction quality is shown in figure 9 (right). Except for the first MART reconstruction process, where several iterations are needed to reach a reconstruction quality that enables a reliable analysis by crosscorrelation, the most prominent improvement is made when $E_{G}$ is evaluated after the results from the two exposures are combined. An additional improvement, comparable to the latter, is obtained after the first MART iteration of the enhanced guess field. This may also suggest that the MTE technique can be implemented in a relatively efficient way, limiting the number of time-consuming MART iterations to a single one or two at most, when the first enhanced objects have already been obtained.

The velocity field between reconstructed $3 \mathrm{D}$ objects is obtained with the volume deformation iterative multigrid technique (VODIM, Scarano et al 2006). In the present case, the problem has been reduced to $2 \mathrm{D}$ objects, which are therefore analyzed with WIDIM (Scarano and Riethmuller 2000) using interrogation windows elongated in the $X$ direction. The window size is chosen in such a way as to obtain, on average, five particles in the interrogation area also at the lower values of $N_{S}$ considered in the simulation. A detail of the velocity field obtained from the object pair reconstructed with MART is shown in figure 11 (top-left) and compared with that resulting from MTE with ten enhancement steps (figure 11, top-right). Although the overall velocity pattern is already well represented for the MART reconstruction, a significant reduction of random errors is obtained with the MTE method.

A detail of the cross-correlation map in a $21 \times 21$ voxel neighborhood of the origin is shown in figure 10 . The comparison between the correlation coefficient obtained from MART reconstructed objects and that from enhanced objects shows the extent of the signal-to-noise improvement obtained by the MTE technique. The position of the peak indicates a displacement of 3 voxels along the $X$-direction; the signal-tonoise ratio is three times higher after ten MTE steps.

The dispersion plot of the precision error of velocity measurement is shown in figure 11 (bottom-left), where $\varepsilon_{w}=w_{R}-w_{0}$ is the difference between the displacement along the depth direction obtained from cross-correlation of the reconstructed objects $\left(w_{R}\right)$ and that obtained from correlation of the original intensity fields $\left(w_{0}\right)$; the same applies for the $u$-component. The standard deviation of the two components of the error (figure 11, bottom-right) increases with the seeding density; values comparable with those obtained applying MART only are reached after ten MTE steps for approximately three times higher seeding density. Conversely, for high values of the seeding density, the measurement precision error is reduced by a factor 3 with the MTE technique. More iterations appear to be required for the $w$-component to obtain the same improvement.

\subsection{Time-resolved recordings}

When the particle motion is measured by a continuous sequence of single exposures in time-resolved mode, recordings are available that allow us to include more than two exposures in the MTE technique for each single object. In 

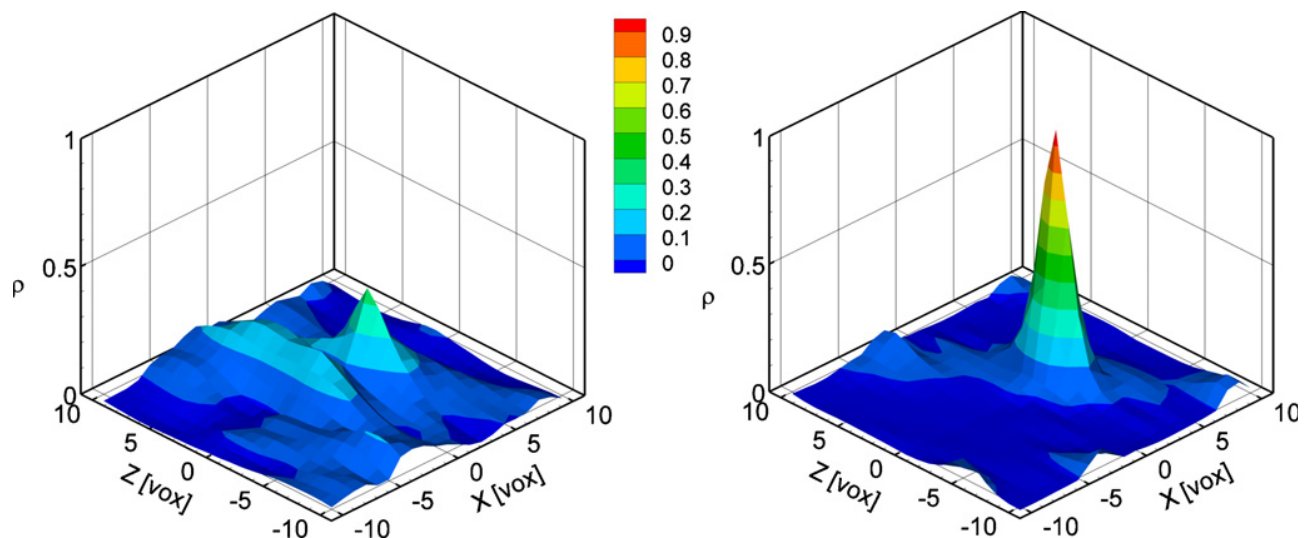

Figure 10. Detail of the cross-correlation map obtained by two reconstructed subsequent exposures at $N_{S}=0.6$. Left: objects reconstructed with MART. Right: enhanced reconstructed objects after ten MTE steps.
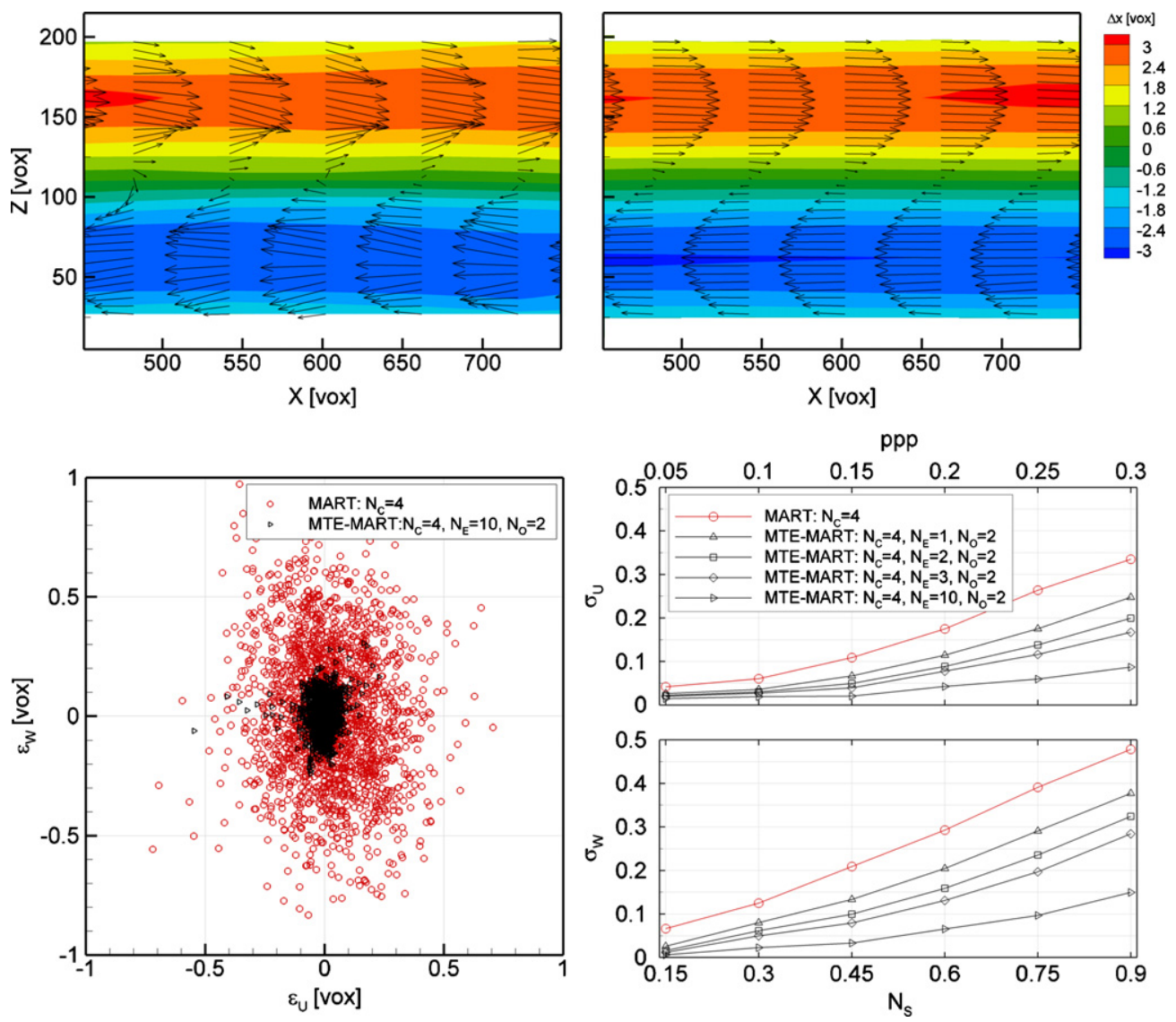

Figure 11. Top-left: displacement field obtained by cross-correlation between two objects reconstructed by MART $\left(N_{S}=0.6\right)$. Top-right: displacement field obtained by cross-correlation between the objects reconstructed using ten steps of MTE-MART. Bottom-left: scatter plot of the difference between the actual and the computed displacement for the two components $U$ and $W$, relative to the displacement field presented in the top row. Bottom-right: standard deviation of the error in the displacement components as a function of $N_{S}(p p p)$ and $N_{E}$.

this case, equation (8) needs to be rewritten in a more general form as

$$
E_{G} \approx E_{C}+\frac{1}{N_{O}}\left(\sum_{n=1}^{N_{O}} E_{I, n}\right),
$$

where $N_{O}$ is the number of exposures taken into account to form the guess. When $N_{O}>2$ important advantages are found:

(1) the rate at which the incoherent (ghost) intensity decays is directly proportional to $N_{O}$;
(2) the asymptotic behavior of the MTE method is expected to be that of a tomographic system with a number of cameras multiplied by $N_{O}$.

The present analysis is carried out for $N_{O}=\{3,5,7\}$ and values beyond 7 are considered unrealistic for real applications. The displacement predictor between the $m$ th and $n$th exposures is given in this case by the sum of the individual displacement fields obtained between subsequent exposures. 

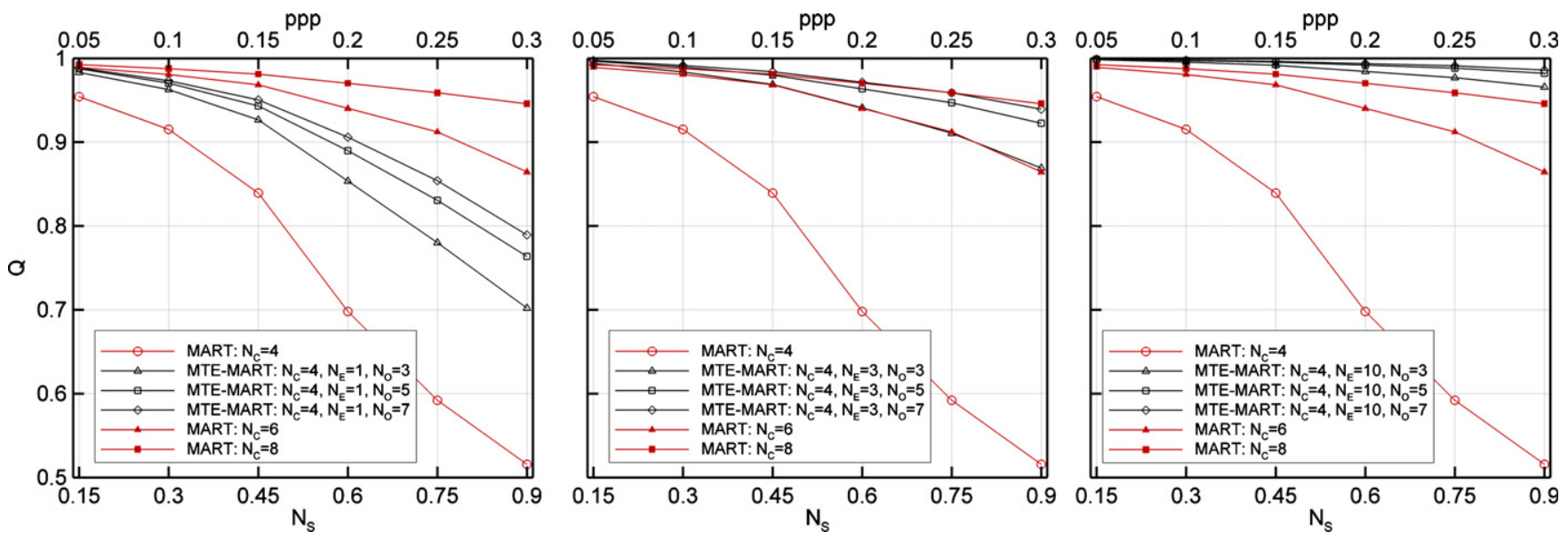

Figure 12. Effect of number of objects $N_{O}$ and enhancement steps $N_{E}$ on the accuracy of reconstruction by MTE-MART.
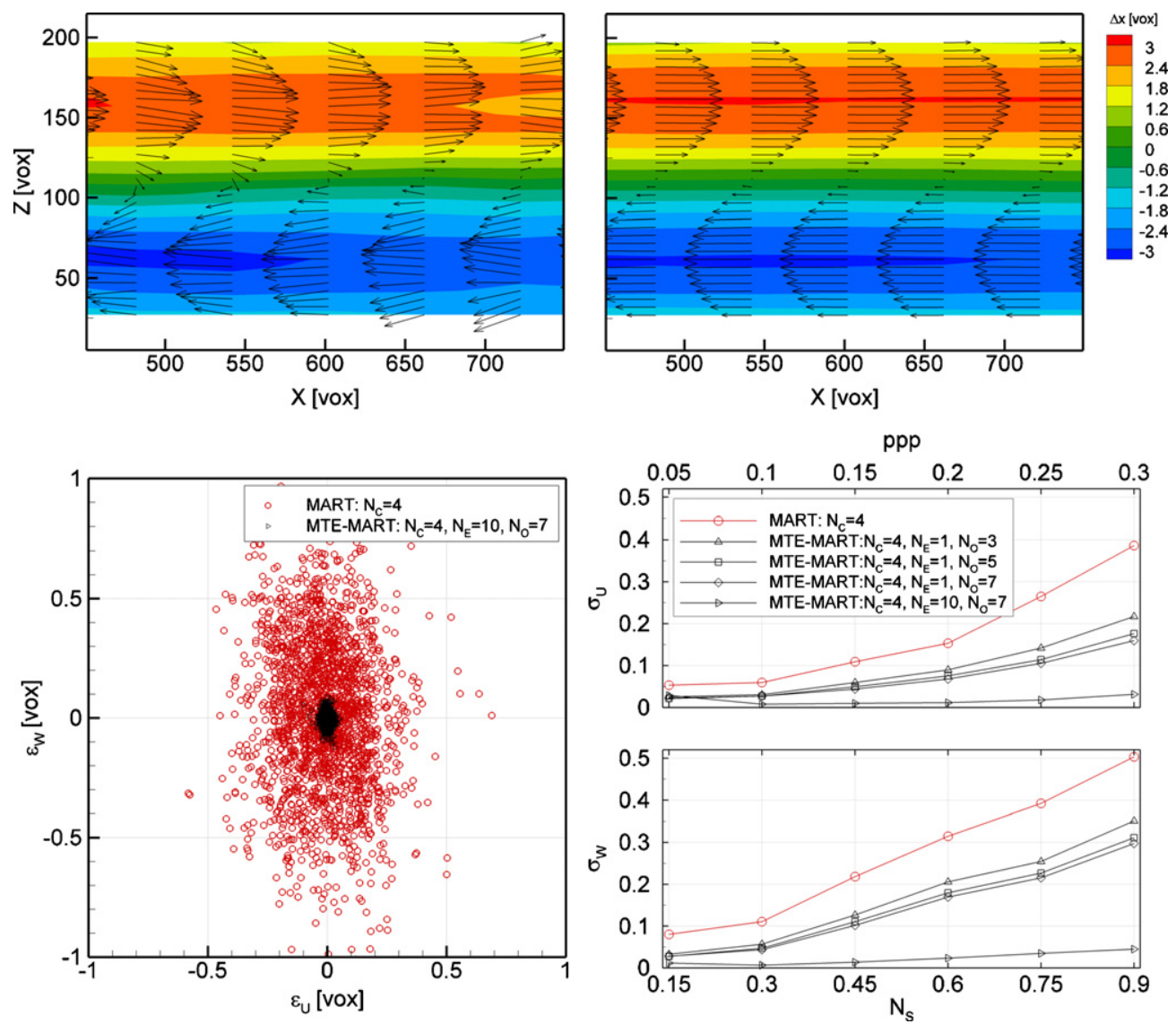

Figure 13. Top: displacement field obtained by cross-correlation between two objects reconstructed by MART (left) and MTE-MART (right) with $N_{E}=10$ and $N_{O}=7\left(N_{S}=0.6\right)$. Bottom-left: 2D scatter plot of the displacement error. Bottom-right: standard deviation $\sigma$ of the error in the displacement components as a function of $N_{S}(p p p)$ varying the values of $N_{E}$ and $N_{O}$.

Figure 12 summarizes the effect of the number of objects $N_{O}$ and that of enhancement steps $N_{E}$ upon the reconstruction accuracy $Q$. The increase in $Q$ with the number of exposures is visible but somehow less pronounced than that obtained with subsequent enhancement steps. For $N_{E}=10$ and $N_{O}>$ 5 , the reconstruction quality becomes almost independent of $N_{S}$ and is always above 0.95 , which confirms the potential of the MTE approach to analyze tomographic PIV recording from highly seeded images. The corresponding velocity field is significantly more regular than that obtained for a single MART analysis, and the error standard deviation reduces to approximately 0.05 voxels with a difference in the convergence rate between $w$ and $u$ that is similar to the case of two exposures (figure 13).

For low values of the seeding density $\left(N_{S}<0.3\right)$, the reduction of precision error is larger than that expected solely for the ghost particles attenuation. This may be ascribed to a slight reduction of discretization errors in that the particles are 


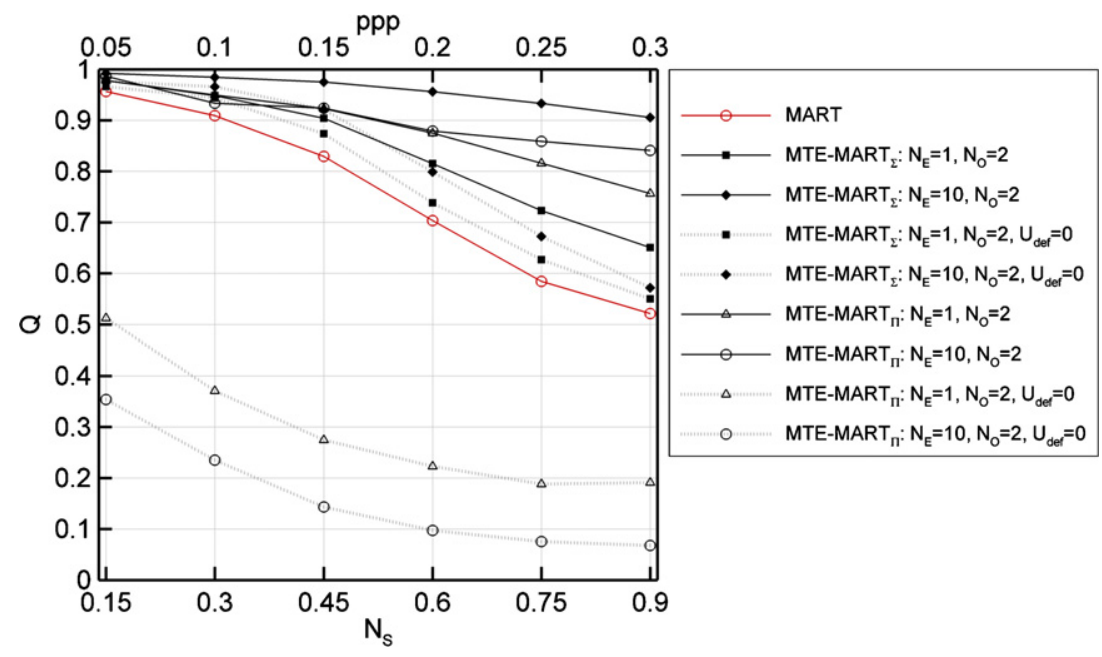

Figure 14. Comparison between the averaging $(\Sigma)$ and multiplicative $(\Pi)$ approach for MTE-MART. Reconstruction quality factor $Q$ as a function of $N_{S}(p p p)$. Results from computed motion field from cross-correlation (black lines) and from an erroneous motion field (dotted lines).
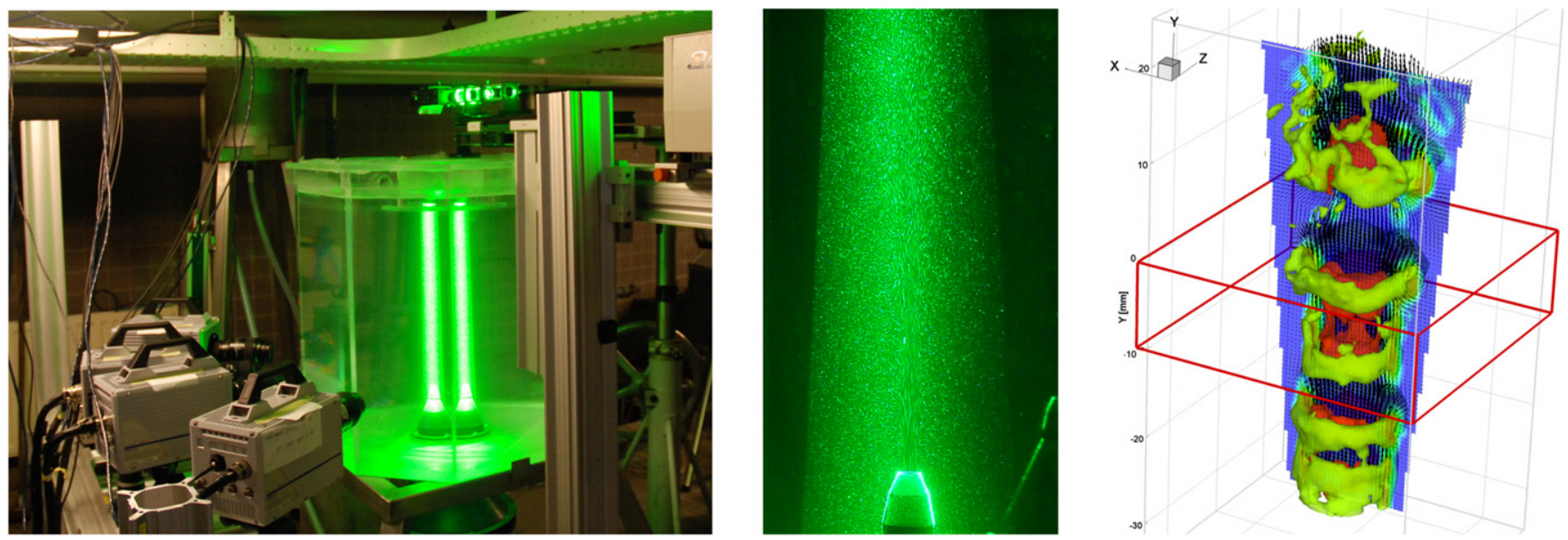

Figure 15. Left: jet tomography facility. Center: planar illumination. Right: instantaneous vorticity magnitude (yellow) and vertical velocity (red) iso-surfaces. Velocity vectors and vorticity magnitude contours in data slice on the symmetry axis.

reconstructed at different sub-voxel locations in each pseudosimultaneous object.

\section{Multiplicative variant of the MTE algorithm}

The MTE technique so far discussed is implemented with an average between the reconstructed object and the PSOs to form the initial guess. The incoherent intensity field due to ghost particles is expected to be reduced in relation to the number of exposures $N_{O}$ used to build the new guess. When doubleframe recordings are available, the ghost intensity can be halved during this procedure. An alternative to the averaging operation is to multiply the reconstructed field and its PSO and raise them to the power $1 / N_{O}$. This approach can lead to a faster suppression of the incoherent intensity by improving the convergence rate of the MTE-MART method. In the following, the MTE method based on the averaging approach (indicated with the symbol $\Sigma$ ) and that based on multiplication (symbol $\Pi$ ) are compared in terms of quality factor $Q$ as a function of the seeding density $N_{S}$ (figure 14). The accuracy of the reconstruction, performed with four cameras, is shown at different enhancement steps $\left(N_{E}=1\right.$ and $\left.N_{E}=10\right)$ for both $\mathrm{MTE}_{\Sigma}$ and $\mathrm{MTE}_{\Pi}$. Two different test cases are analyzed: in the first case (solid lines) the velocity field between the exposures is computed by means of cross-correlation; instead, in the second one (dotted lines), the velocity field is arbitrarily set to zero. The latter is intended to verify the robustness of the method with respect to errors (e.g. spurious vectors) in the displacement predictor field. For an accurate displacement field, the first step of $\mathrm{MTE}_{\Pi}$ largely improves the result and is comparable to two steps of $\mathrm{MTE}_{\Sigma}$. The iterative use of $\mathrm{MTE}_{\Pi}$ does not maintain the same trend: after ten steps, actually, the $\mathrm{MTE}_{\Sigma}$ appears to provide a more accurate reconstruction. Both methods, however, attain more accurate object reconstruction compared to MART.

Instead, when the velocity field used in the deformation procedure $\left(\boldsymbol{V}_{\text {pred }}\right)$ is set to zero, $\mathrm{MTE}_{\Pi}$ becomes very inaccurate with reconstruction accuracy more than halved compared to MART. Instead, for the same condition $\left(\boldsymbol{V}_{\text {pred }}=\right.$ 0) $\mathrm{MTE}_{\Sigma}$ basically maintains the same accuracy as MART. 

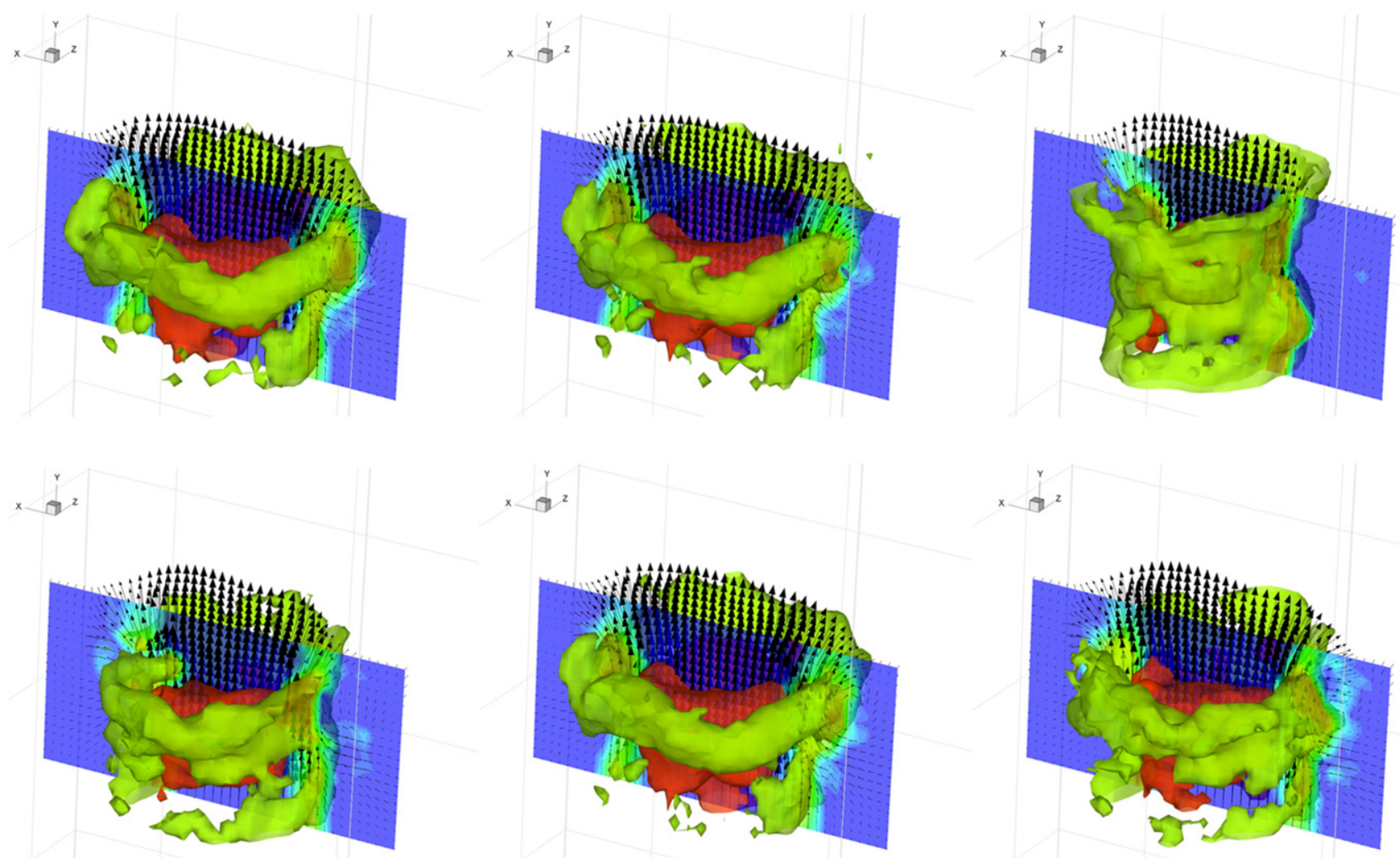

Figure 16. Detail of the flow around a vortex ring. Top-left $N_{C}=4, N_{E}=0$; top-center $N_{C}=3, N_{E}=0$; top-right $N_{C}=2, N_{E}=0$. Bottom-left $N_{C}=2, N_{E}=5$; bottom-center $N_{C}=3, N_{E}=5$; bottom-right $N_{C}=2, N_{E}=35$.

The undesired behavior of $\mathrm{MTE}_{\Pi}$ can be explained recalling the discussion in section 3: the multiplication approach leads to cancellation of the coherent part of the reconstruction (actual particles) if they are not simultaneously present at the same location in the reconstructed object and the PSO. Instead, the averaging approach maintains at least the part of the coherent intensity coming from the reconstructed object. In conclusion, it can be stated that the $\mathrm{MTE}_{\Sigma}$ algorithm or briefly MTE-MART candidates best for an accurate and reliable reconstruction and the multiplicative approach may only be considered useful if the displacement field is known with a high level of reliability.

\section{Transitional jet experiment}

The MTE-MART method applicability to real experiments is verified by means of a tomographic PIV experiment conducted in the jet tomography facility (JTF) of TU Delft. The flow facility is designed for jet noise research and allows full optical access (figure 15, left). The transition patterns in circular jets with laminar flow at the exit in the Reynolds number range 2500 to 22000 are investigated with emphasis on 3D instabilities occurring at the core breakdown. The jet is issued by a contoured nozzle following the design of Schram (2003) with a contraction ratio of 53:1 and a $10 \mathrm{~mm}$ exit diameter. An octagonal tank with flat Perspex walls of $60 \mathrm{~cm}$ diameter and $80 \mathrm{~cm}$ height confines the jet issued at the bottom wall. The flow is seeded with $56 \mu \mathrm{m}$ polyamide particles at a concentration of approximately 0.7 particles $\mathrm{mm}^{-3}$. The light source is a Quantronix Darwin-Duo Nd:YLF laser $(2 \times$ $25 \mathrm{~mJ} /$ pulse@ $1 \mathrm{kHz}$ ). The initial light beam diameter of $6 \mathrm{~mm}$ is expanded to $30 \mathrm{~mm}$ by a Linos beam expander. The volume illumination over a cylinder is optimal for this flow configuration in that the lines of sight intersection with the illuminated volume have variable length with a maximum at the jet axis and decreasing with radial distance. The tomographic imaging system is composed of four Photron Fastcam SA1 CMOS cameras $(1024 \times 1024$ pixels, $5400 \mathrm{fps})$ equipped with Nikon objectives $(f=105 \mathrm{~mm})$ set at $f \#=32$ to obtain focused particle images along the entire depth of the illuminated volume. An optical magnification $M=0.4$ yields a field of view of approximately $50 \times 50 \mathrm{~mm}^{2}$ and a digital resolution of 20 pixels $\mathrm{mm}^{-1}$. The measurement domain extends from the jet exit up to five diameters downstream and encompasses three diameters in the radial direction. Illumination and imaging synchronization at $1 \mathrm{kHz}$ is provided by a LaVision high-speed controller, and the data acquisition and processing are performed with DaVis7.2 on a PC equipped with INTEL dual quad-core $2.66 \mathrm{GHz}$ CPU and $3 \mathrm{~GB}$ RAM memory. The tomographic data analysis is performed with a custom version of DaVis where MTE has been implemented for double exposures $\left(N_{O}=2\right)$.

The tomographic PIV system is operated at conditions $\left(N_{S}<0.3\right.$, ppp $\left.<0.05\right)$ such that the four-camera system yields results to be considered as reference data. The timeresolved measurement by four cameras shows that the velocity 


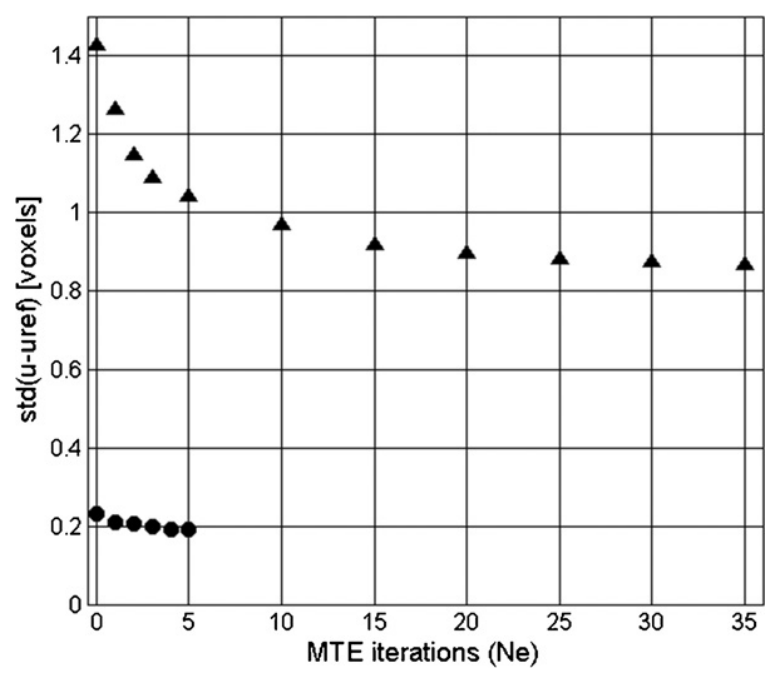

Figure 17. Measured velocity difference relative to the reference case $\left(N_{C}=4\right)$. Two- $(\boldsymbol{\Delta})$ and three-camera $(\bullet)$ system.

measured in potential flow regions yields a noise level below 0.03 voxels $\left(0.5 \% V_{\text {jet }}\right)$.

The verification of the MTE method is performed comparing the measurement obtained with two-camera and three-camera systems to the reference data obtained with four cameras. The number of MTE iterations $\left(N_{E}\right)$ is varied as parameter. Except for the first MART reconstruction where five iterations are applied, all subsequent MTE steps make use of only two MART iterations.

The jet undergoes first Kelvin-Helmholtz-like instability with the formation of vortex rings, which subsequently pair (leap-frogging). Further away from the exit $(Y / D>4)$, the transition to the $3 \mathrm{D}$ regime is observed first with azimuthal wiggles in the vortex rings and then with the formation of axial structures.

A sub-region of the flow where a quasi axi-symmetric vortex ring is formed is considered for the present assessment (red box in figure 15, right). The flow structure is captured reliably for $N_{C}=4$ with a spatial resolution of approximately 5 vectors across the vortex core. The result obtained using fewer cameras on the same images is shown in figure 16 . When $N_{C}$ $=3$, the jet flow structure compares satisfactorily with the reference although the noise level is slightly increased in the vorticity iso-surface. For $N_{C}=2$, the global flow structure is profoundly distorted with local errors approaching $100 \% V_{\text {jet }}$ (the particle images displacement at jet exit is approximately 7 voxels). The jet cross section is elongated along one of the viewing directions indicating that the ghost particle signal dominates over that of the actual particles.

The application of five MTE iterations to the three-camera reconstruction yields a result closer to the reference data with a slightly more regular pattern of the vorticity iso-surface. Nevertheless, because the three-camera reconstruction is already rather close to the reference data, the improvement in this case is marginal and the measurement error is expected to be dominated by the cross-correlation analysis rather than ghost particle intensity. The reconstruction with two cameras, instead, is significantly improved after five MTE iterations (figure 16, bottom-left): the core of the ring vortex becomes visible and the velocity vectors recover a circulatory pattern. The situation is further improved when the MTE is repeated up to 35 iterations: the vortex ring becomes distinct from the jet shear layer beneath it and also the pattern of velocity vectors recovers a more regular shape resembling the reference data.

A quantitative assessment of the data enhancement obtained by MTE on the present experiment is obtained by

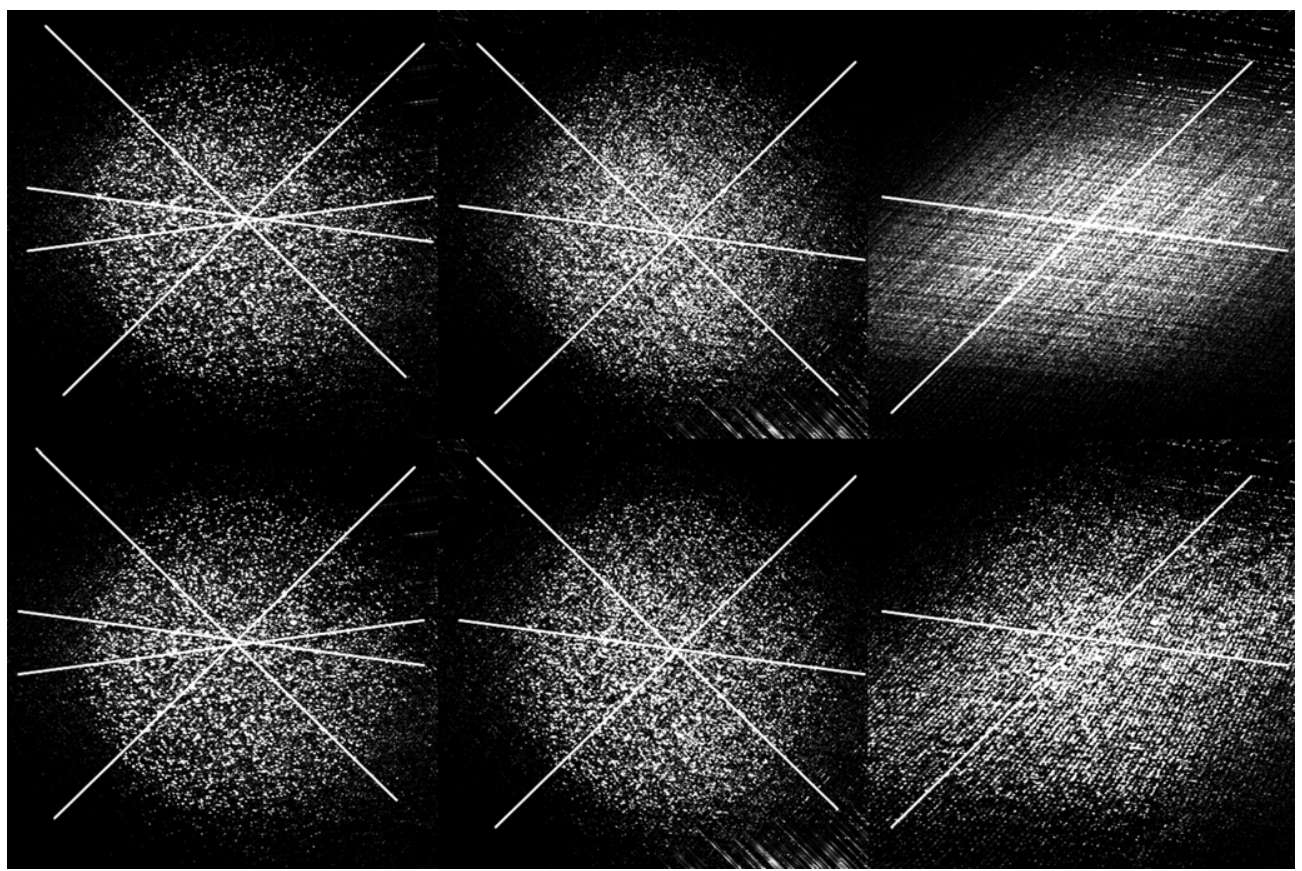

Figure 18. Reconstructed light intensity distribution (viewing directions indicated by white lines). Top-left $N_{C}=4, N_{E}=0$; top-center $N_{C}=3, N_{E}=0$; top-right $N_{C}=2, N_{E}=0$. Bottom-left $N_{C}=4, N_{E}=1 ;$ bottom-center $N_{C}=3, N_{E}=5 ;$ bottom-right $N_{C}=2, N_{E}=10$. 
comparing the local velocity vector components measured from two and three cameras with the measurement obtained by four cameras. The standard deviation of the difference is taken as the norm of the error. The results given in figure 17 illustrate that for $N_{C}=3$, the result is already close to the reference data $\left(\varepsilon_{V} \sim 0.2\right.$ voxels). The error is slightly reduced in the first five MTE iterations. The improvement is more pronounced for the two-camera system where the estimated error is almost halved, which is consistent with the data inspection in figure 16 .

The accuracy of the reconstructed light intensity is inspected by a projected view of the object onto a plane perpendicular to the jet axis illustrated in figure 18. The results from the MART reconstruction are given in the upper row (four, three and two cameras from left to right). The case with four cameras is considered as reference and will be used for the evaluation of the relative quality factor $Q_{R}$. The white lines indicate the viewing directions of the active cameras. Adding one or two MTE steps adds very little to the four-camera system (bottom-left). In terms of relative quality factor, these two objects differ by less than $1 \%$. The effect is instead more pronounced for the three-camera system. The MART reconstruction yields $Q_{R}=0.73$ and after five MTE iterations, $Q_{R}$ increases to 0.80 . Finally, the two-camera system is clearly inadequate to reconstruct the intensity field, with the MART reconstruction yielding as low $Q_{R}$ as 0.25 . After more than ten iterations, the value increases to 0.31 , which is still deemed insufficient for a reliable cross-correlation analysis. Nevertheless, a higher definition of the object is attained.

\section{Conclusions}

A novel technique for the enhancement of the MART reconstruction in Tomo-PIV has been introduced. The theoretical background and working principle of the motion tracking enhancement (MTE) have been discussed and its performances have been assessed by means of twodimensional simulations of the reconstruction of both doubleframe and time-resolved recordings. The accuracy of the reconstruction, evaluated through the $Q$ factor, returned the most significant increase for seeding density approximately three times larger than those reported in the literature for double-frame recordings. When time-resolved data are available, the accuracy of the reconstruction appears to further increase in relation to the number of exposures employed. The displacement fields obtained by spatial cross-correlation of reconstructed objects after enhancement have been analyzed showing a decrease of the precision error of approximately three times for double-frame and almost ten times for multiple recordings. The robustness of the MTE algorithm with respect to large errors in the measurement has been verified, with the conclusion that the present approach returns in the worst case a result comparable to simple MART. Instead the potentially faster approach by multiplication of object and pseudo-simultaneous objects is extremely sensitive to errors and rapidly degrades the result.

The application to real experiments performed with a time-resolved tomographic PIV system show that the reconstruction accuracy from a lowered number of cameras (three in this case) can be brought close to that of a larger number of cameras with the MTE approach (operated in double-frame mode). Moreover, a reduction in the velocity measurement uncertainty is observed for systems with two and three cameras, which is consistent with the trend inferred by the numerical assessment.

The overall effect of the MTE-MART method is comparable to that of increasing the number of viewing cameras: the asymptotic behavior provides comparable reconstruction accuracy to that obtained by doubling the number of cameras with MART. According to the current analysis, the MTE technique could enable Tomo-PIV experiments with seeding particle concentration two to three times higher than that currently practiced (50000 particles/Mpixel) possibly leading to experiments with more than 100000 particles/Mpixel.

Given its iterative structure, the MTE technique is computationally more intensive than the data processing currently practiced for Tomo-PIV. Further investigation about the computational time needed is also required in order to improve the efficiency of the numerical evaluation of MTEMART.

\section{Acknowledgments}

This work is carried out in the frame of the FLOVIST project (Flow Visualization Inspired Aeroacoustics with Time Resolved Tomographic Particle Image Velocimetry), funded by the European Research Council (ERC) grant no 202887. KJB acknowledges support from the Fund for Scientific Research-Flanders. Dirk Michaelis (LaVision $\mathrm{GmbH}$ ) is acknowledged for the support on the implementation of MTE in the DaVis software.

\section{References}

Atkinson C H, Dillon-Gibbons C J, Herpin S and Soria J 2008 Reconstruction techniques for tomographic PIV (Tomo-PIV) of a turbulent boundary layer 14th Int. Symp. on Applications of Laser Techniques to Fluid Mechanics (7-10 July, Lisbon, Portugal)

Atkinson C and Soria J 2009 An efficient simultaneous reconstruction technique for tomographic particle image velocimetry Exp. Fluids $\mathbf{4 7}$ 553-68

Brücker C H 1995 Digital-particle-image-velocimetry (DPIV) in a scanning light sheet: 3D starting flow around a short cylinder Exp. Fluids 19 255-63

Coëtmellec S, Buraga-Lefebvre C, Lebrun D and Özkul C 2001 Application of in-line digital holography to multiple plane velocimetry Meas. Sci. Technol. 12 1392-97

Elsinga G E 2008 Tomographic particle image velocimetry $P h D$ Thesis Aerospace Engineering Department, Delft University of Technology

Elsinga G E, Scarano F, Wieneke B and van Oudheusden B 2006a Tomographic particle image velocimetry Exp. Fluids 41 933-47

Elsinga G E, van Oudheusden B W and Scarano F 2006b Experimental assessment of tomographic PIV accuracy Int. Symp. on Applications of Laser Techniques to Fluid Mechanics (26-29 June, Lisbon, Portugal) 
Elsinga G E, Westerweel J, Scarano F and Novara M 2009 On the velocity of ghost particles 8 th Int. Symp. on Particle Image Velocimetry (25-28 August, Melbourne, Victoria, Australia)

Elsinga G E, Wieneke B, Scarano F and Schröder A 2008 Tomographic 3D-PIV and applications Particle Image Velocimetry-New Developments and Recent Applications (Topics in Applied Physics vol 112) ed A Schröder and C Willert (Berlin: Springer)

Herman G T and Lent A 1976 Iterative reconstruction algorithms Comput. Biol. Med. 6 273-94

Hinsch K D 2002 Holographic particle image velocimetry Meas. Sci. Technol. 13 R61-72

Keane R D and Adrian R J 1990 Optimization of particle image velocimeters: part I. Double pulsed systems Meas. Sci. Technol. 1 1202-15

Maas H G, Gruen A and Papantoniou D 1993 Particle tracking velocimetry in three-dimensional flows Exp. Fluids 15 133-46

Mishra D, Muralidhar K and Munshi P 1999 A robust MART algorithm for tomographic applications Numer. Heat Transfer 35 485-506

Pereira F and Gharib M 2001 Defocusing digital particle image velocimetry and the three-dimensional characterization of two-phase flows Meas. Sci. Technol. 13 683-94

Petra S, Schröder A and Schnörr C 2009 3D tomography from few projections in experimental fluid dynamics Imaging Measurement Methods (NNFM vol 106) (Berlin: Springer) pp 63-72

Scarano F, Elsinga G E, Bocci E and Van Oudheusden B W 2006 Investigation of 3-D coherent structures in the turbulent cylinder wake using Tomo-PIV 13th Int. Symp. on Applications of Laser Techniques to Fluid Mechanics (26-29 June, Lisbon, Portugal)

Scarano F and Poelma C 2009 Three-dimensional vorticity patterns of cylinder wakes Exp. Fluids 47 69-83

Scarano F and Riethmuller M L 2000 Advances in iterative multigrid PIV image processing Exp. Fluids Suppl S51-60

Schram C 2003 Aeroacoustics of subsonic jets: prediction of the sound produced by vortex pairing based on particle image velocimetry $P h D$ Thesis Technische Universiteit Eindhoven

Schröder A, Geisler R, Elsinga G E, Scarano F and Dierksheide U 2008 Investigation of a turbulent spot and a tripped turbulent boundary layer flow using time-resolved tomographic PIV Exp. Fluids 44 305-16

Schröder A, Geisler R, Sieverling A, Wieneke B, Henning A, Scarano F, Elsinga G E and Poelma C 2009 Lagrangian aspects of coherent structures in a turbulent boundary layer flow using TR-Tomo PIV and PTV 8th Int. Symp. on Particle Image Velocimetry (25-28 August, Melbourne, Victoria, Australia)

Verhoeven D 1993 Limited-data computed tomography for the physical sciences Appl. Opt. 32 3736-54

Wieneke B 2008 Volume self-calibration for 3D particle image velocimetry Exp. Fluids $\mathbf{4 5} 549-56$

Wieneke B and Taylor S 2006 Fat-sheet PIV with computation of full 3D-strain tensor using tomographic reconstruction 13th Int. Symp. on Applications of Laser Techniques to Fluid Mechanics (26-29 June, Lisbon, Portugal)

Worth N A and Nickels T B 2008 Acceleration of Tomo-PIV by estimating the initial volume intensity distribution Exp. Fluids $45847-56$ 